\title{
Measurement of the top quark pair production cross section in dilepton final states containing one $\tau$ lepton in pp collisions at $\sqrt{s}=13 \mathrm{TeV}$
}

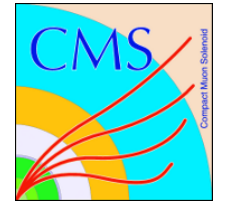

\section{The CMS collaboration}

E-mail: cms-publication-committee-chair@cern.ch

ABSTRACT: The cross section of top quark pair production is measured in the t $\bar{t} \rightarrow$ $\left(\ell \nu_{\ell}\right)\left(\tau_{\mathrm{h}} \nu_{\tau}\right) \mathrm{b} \overline{\mathrm{b}}$ final state, where $\tau_{\mathrm{h}}$ refers to the hadronic decays of the $\tau$ lepton, and $\ell$ is either an electron or a muon. The data sample corresponds to an integrated luminosity of $35.9 \mathrm{fb}^{-1}$ collected in proton-proton collisions at $\sqrt{s}=13 \mathrm{TeV}$ with the CMS detector. The measured cross section is $\sigma_{\mathrm{t} \overline{\mathrm{t}}}=781 \pm 7$ (stat) \pm 62 (syst) \pm 20 (lumi) pb, and the ratio of the partial width $\Gamma\left(\mathrm{t} \rightarrow \tau \nu_{\tau} \mathrm{b}\right)$ to the total decay width of the top quark is measured

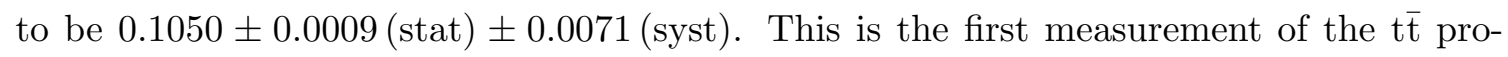
duction cross section in proton-proton collisions at $\sqrt{s}=13 \mathrm{TeV}$ that explicitly includes $\tau$ leptons. The ratio of the cross sections in the $\ell \tau_{\mathrm{h}}$ and $\ell \ell$ final states yields a value $R_{\ell \tau_{\mathrm{h}} / \ell \ell}=0.973 \pm 0.009$ (stat) \pm 0.066 (syst), consistent with lepton universality.

KEYwoRDS: Hadron-Hadron scattering (experiments), Top physics

ArXiv EPRINT: 1911.13204 


\section{Contents}

1 Introduction 1

2 The CMS detector 2

3 Event simulation $\quad 2$

4 Event reconstruction and selection 3

5 Event categories and fit procedure $\quad 5$

$\begin{array}{lll}6 & \text { Background estimate } & 7\end{array}$

$\begin{array}{llr}7 & \text { Systematic uncertainties } & 8\end{array}$

8 Results 10

9 Summary 14

$\begin{array}{ll}\text { The CMS collaboration } & 21\end{array}$

\section{Introduction}

In proton-proton (pp) collisions at the CERN LHC, top quarks are produced mainly in pairs ( $\mathrm{t} \overline{\mathrm{t}})$ and subsequently decay to $\mathrm{b}$ quarks and $\mathrm{W}$ bosons: $\mathrm{pp} \rightarrow \mathrm{t} \overline{\mathrm{t}} \rightarrow \mathrm{W}^{+} \mathrm{bW} \mathrm{W}^{-} \overline{\mathrm{b}}$. The decay modes of the two $\mathrm{W}$ bosons determine the observed event signature. The dilepton decay channel denotes the case where both $\mathrm{W}$ bosons decay leptonically. In this paper, we consider the process $\mathrm{t} \overline{\mathrm{t}} \rightarrow\left(\ell v_{\ell}\right)\left(\tau v_{\tau}\right) \mathrm{b} \overline{\mathrm{b}}$, where one $\mathrm{W}$ boson decays into $\ell v_{\ell}$ where $\ell$ is either an electron $(\mathrm{e})$ or a muon $(\mu)$, and the other into a tau lepton and a neutrino $\left(\tau v_{\tau}\right)$. The expected fraction of events in this final state corresponds to $\approx 4 / 81(\approx 5 \%)$ of all $\mathrm{t} \overline{\mathrm{t}}$ decays, i.e. equivalent to the fraction of all light dilepton channels $(e e, \mu \mu, \mathrm{e} \mu)$.

Recent checks of lepton flavour universality violation [1-8] sparked a renewed interest towards measurements involving $\tau$ leptons, owing to a potential disagreement with standard model (SM) predictions. The $t \rightarrow\left(\tau v_{\tau}\right)$ b decay exclusively involves third-generation leptons and quarks which, owing to their large masses, may be particularly sensitive to beyond SM contributions. For example, the existence of a charged Higgs boson [9-12] may give rise to anomalous $\tau$ lepton production that could be observed in this decay channel.

This is the first measurement of the $t \bar{t}$ production cross section in pp collisions at $\sqrt{s}=13 \mathrm{TeV}$ that explicitly includes $\tau$ leptons. The data sample was collected in 2016 with the CMS detector at the LHC and corresponds to an integrated luminosity of $35.9 \mathrm{fb}^{-1}$. The $\tau$ lepton is identified through its visible decay products, either hadrons $\left(\tau_{\mathrm{h}}\right)$ or leptons 
$\left(\tau_{\ell}\right)$, with the corresponding branching fractions $\mathcal{B}\left(\tau_{\mathrm{h}} \rightarrow\right.$ hadrons $\left.+v_{\tau}\right) \approx 65 \%$ and $\mathcal{B}\left(\tau_{\ell} \rightarrow\right.$ $\left.\ell v_{\ell} v_{\tau}\right) \approx 35 \%$. In the first case, the $\tau_{\mathrm{h}}$ decays into a narrow jet with a distinct signature, whereas the leptonic decays are difficult to distinguish from prompt electron or muon production. In this measurement, the signal includes only $\tau$ leptons that decay hadronically, and $\ell$ does not include leptons from $\tau$ decays. The dominant background contribution comes from events where a jet is misidentified as a $\tau_{\mathrm{h}}$, mostly from $\mathrm{t} \overline{\mathrm{t}}$ lepton + jets events, i.e. $t \bar{t} \rightarrow\left(\ell v_{\ell}\right)\left(q \bar{q}^{\prime}\right) b \bar{b}$. The cross section is measured by performing a profile likelihood ratio (PLR) fit [13] to the transverse mass of the system containing the lepton (e or $\mu$ ) and the missing transverse momentum, in two kinematic categories of the selected events for each of the $\mathrm{e} \tau_{\mathrm{h}}$ and $\mu \tau_{\mathrm{h}}$ final states. The cross section is measured in the fiducial phase space of the detector and also extrapolated to the full phase space. The ratio of the cross sections in the $\ell \tau$ and light dilepton [14] final states $\sigma_{\mathrm{t} \overline{\mathrm{t}}}(\ell \tau) / \sigma_{\mathrm{t} \overline{\mathrm{t}}}(\ell \ell)$, and the ratio of the partial to the total decay width of the top quark $\Gamma\left(\mathrm{t} \rightarrow \tau \nu_{\tau} \mathrm{b}\right) / \Gamma_{\text {total }}$ are evaluated.

This paper is organized as follows: the CMS detector layout is briefly described in section 2; details about the simulated event samples used in the data analysis are provided in section 3; section 4 covers the event reconstruction and the event selection; the event categorization and the fit procedures are described in section 5 ; the background determination procedure is given in section 6 ; the description of the systematic uncertainties is presented in section 7; measurements of the cross sections, and the ratio of the partial to the total $\mathrm{t} \overline{\mathrm{t}}$ decay width are discussed in section 8; and the results are summarized in section 9.

\section{The CMS detector}

The central feature of the CMS apparatus is a superconducting solenoid of $6 \mathrm{~m}$ internal diameter, providing a magnetic field of $3.8 \mathrm{~T}$. Within the solenoid volume are a silicon pixel and strip tracker, a lead tungstate crystal electromagnetic calorimeter, and a brass and scintillator hadron calorimeter, each composed of a barrel and two endcap sections, covering $0<\varphi<2 \pi$ in azimuth and $|\eta|<2.5$ in pseudorapidity. Forward calorimeters extend the pseudorapidity coverage provided by the barrel and endcap detectors. Muons are detected in gas-ionization chambers embedded in the steel flux-return yoke outside the solenoid. The detector is nearly hermetic, providing reliable measurement of the momentum imbalance in the plane transverse to the beams. A two-level trigger system [15] selects the most interesting pp collision events for use in physics analysis. A more detailed description of the CMS detector, together with a definition of the coordinate system used and the relevant kinematic variables, can be found in ref. [16].

\section{Event simulation}

The analysis makes use of simulated samples of $t \bar{t}$ events, as well as other processes that result in reconstructed $\tau$ leptons in the final state. These samples are used to design the event selection, to calculate the acceptance for t $\bar{t}$ events, and to estimate most of the backgrounds in the analysis. 
Signal te events are simulated with the POWHEG event generator (v2) [17-21] at next-to-leading-order (NLO) accuracy in quantum chromodynamics (QCD). The parton showers are modelled using PYTHIA (v8.2) [22] with the CUETP8M2T4 underlying event (UE) tune [23]. The background samples used in the measurement of the cross section are simulated with POWHEG and MadGraph5_amC@NLO (v2.2.2) [24]. The MADGRAPH5_aMC@NLO generator with MLM matching [25] is used for the simulation of W boson production in association with jets $(\mathrm{W}+$ jets), and Drell-Yan (DY) production in association with jets at leading-order (LO) accuracy. Here, only the leptonic decays of DY events and $\mathrm{W}$ bosons are simulated, and up to four additional jets are included. The diboson processes are produced with NLO accuracy: WW with POWHEG, WZ and ZZ with MAdGraph5_amC@NLO with FxFx matching [26]. The POWHEG generator is used for the simulation of $t$-channel single top quark production and single top quark production associated with a W boson (tW) [27, 28]. The single top quark $s$-channel sample is produced with MAdGraPh5_amC@NLO at NLO accuracy with FxFx matching scheme. The simulated events are produced with a top quark mass of $m_{\mathrm{t}}=172.5 \mathrm{GeV}$. The generated events are subsequently processed with PYTHIA using the underlying event tune CUETP8M1 to provide the showering of the partons, and to perform the matching of the soft radiation with the contributions from direct emissions included in the matrix-element (ME) calculations. The default parton distribution functions (PDFs) are the NNPDF3.0 [29]. The $\tau$ decays are simulated with PYTHIA, which correctly accounts for the $\tau$ lepton polarization in describing the kinematic properties of the decay. The CMS detector response is simulated with Geant4 [30]. Additional pp interactions in the same or nearby bunch crossings (pileup, PU) are superimposed on the hard collision. Simulated events are reweighted to match the distribution of the number of pileup collisions per event in data. This distribution is derived from the instantaneous luminosity and the inelastic cross section [31].

The next-to-next-to-leading-order (NNLO) expected SM t $\overline{\mathrm{t}}$ pair production cross section of $832_{-29}^{+20}($ scale $) \pm 35\left(\mathrm{PDF}+\alpha_{S}\right) \mathrm{pb}[32]\left(m_{\mathrm{t}}=172.5 \mathrm{GeV}\right)$ is used for the normalization of the number of $t \bar{t}$ events in the simulation. The first uncertainty includes the uncertainties in the factorization and renormalization scales, while the second is associated with possible choices of PDFs and the value of the strong coupling constant $\left(\alpha_{S}\right)$. The proton structure is described by the CT14 (NNLO) PDF set with the corresponding PDF and $\alpha_{S}$ uncertainties [33]. The $\mathrm{W}+$ jets and $\mathrm{DY}+$ jets backgrounds are normalized to their NNLO cross sections calculated with FEWZ (v3.1) [34]. The $t$-channel and the $s$-channel single top quark production are normalized to the NLO calculations obtained from HATHOR (v2.1) [35, 36]. The production of tW is normalized to the NNLO calculation [37, 38]. Finally, the production of diboson pairs is normalized to the NLO cross section prediction calculated with MCFM $[39,40]$ (v7.0).

\section{Event reconstruction and selection}

The signal event topology is defined by the presence of two b quark jets from the top quark decays, one $\mathrm{W}$ boson decaying leptonically into ev or $\mu \nu$, and a second $\mathrm{W}$ boson decaying into $\tau_{\mathrm{h}} \nu$. In each event, all objects are reconstructed with a particle-flow (PF) 
algorithm [41]. This algorithm combines the information from all subdetectors to identify and reconstruct all types of particles in the event, namely charged and neutral hadrons, photons, muons, and electrons, together referred to as PF objects. These objects are used to construct a variety of higher-level objects and observables, including jets and missing transverse momentum $\left(\vec{p}_{\mathrm{T}}^{\text {miss }}\right)$, which is the negative vector sum of transverse momenta of all reconstructed PF objects. Parameters of jets and the tracks associated with jets provide input variables for $\mathrm{b}$ tagging discriminators. The reconstructed vertex with the largest value of summed physics-object $p_{\mathrm{T}}^{2}$ is taken to be the primary pp interaction vertex. Jets are reconstructed by clustering PF objects with the anti- $k_{\mathrm{T}}$ [42] jet algorithm with a distance parameter $R=0.4$.

Electron or muon candidates are required to originate from the primary vertex, pass quality selection criteria, and be isolated relative to other activity in the event. The relative isolation is based on PF objects within a cone of $\Delta R=\sqrt{(\Delta \eta)^{2}+(\Delta \varphi)^{2}}=0.4$ around the electron or muon track, and defined as $I_{\text {rel }}=\left(E_{\mathrm{ch}}+E_{\mathrm{nh}}+E_{\mathrm{ph}}-0.5 E_{\mathrm{ch}}^{\mathrm{PU}}\right) / p_{\mathrm{T}}$, where $E_{\text {ch }}$ is the transverse energy deposited by charged hadrons from the primary vertex, $E_{\text {nh }}$ and $E_{\mathrm{ph}}$ are the respective transverse energies of the neutral hadrons and photons, and $0.5 E_{\mathrm{ch}}^{\mathrm{PU}}$ is the estimation of the contribution of neutral particles from pileup vertices, calculated as half of the energy of the charged particles from pileup; $p_{\mathrm{T}}$ is the electron or muon transverse momentum. Electron candidates with $I_{\text {rel }}<0.0588$ in the barrel or $I_{\text {rel }}<0.0571$ in the endcaps are considered isolated. The muon candidate is isolated if $I_{\text {rel }}<0.15$ in either the barrel or the endcaps. The lepton isolation requirements are used to suppress backgrounds from multijet production. The charge misidentification probability for electrons and muons is less than $0.5 \%$ and $0.1 \%$, respectively, and is measured from $\mathrm{Z}$ boson decays and simulation [43-45].

Hadronic $\tau$ lepton decays are reconstructed with the hadron-plus-strips (HPS) algorithm [46], which starts from reconstructed jets. In each jet, a charged hadron is combined with other nearby charged hadrons or photons to identify the decay modes. The identification of $\pi^{0}$ mesons is enhanced by clustering electrons and photons in "strips" along the track bending direction to take into account possible broadening of calorimeter signatures by early showering photons. The $\tau_{\mathrm{h}}$ candidates are selected from the following combinations of charged hadrons and strips that correspond to the $\tau$ decay modes: single hadron, hadron plus a strip, hadron plus two strips, and three hadrons. A multivariate analysis of these HPS $\tau_{\mathrm{h}}$ candidates is used to reduce the contamination from quark and gluon jets. A boosted decision tree is trained using a sample of DY events with $\tau_{\mathrm{h}}$ decays as signal and a sample of QCD multijet events as background, both from simulation. Input variables include the multiplicity and the transverse momenta of electron and photon candidates in the vicinity of the $\tau_{\mathrm{h}}$, the kinematic properties of hadrons and strips, and the $\tau_{\mathrm{h}}$ lifetime information, such as the impact parameter of the leading track and the significance of the length of flight to the secondary vertex of the $\tau_{\mathrm{h}}$ candidates with three charged hadrons. Additional requirements are applied to discriminate genuine $\tau_{\mathrm{h}}$ leptons from prompt electrons and muons. The $\tau_{\mathrm{h}}$ charge is taken as the sum of the charges of the corresponding charged hadrons. The misidentification probability for the charge is less than $1 \%$ and it is estimated from $\mathrm{Z} \rightarrow \tau \tau \rightarrow \mu \tau_{\mathrm{h}}$ data events with same-charge $\mu$ and $\tau_{\mathrm{h}}$. The $\tau_{\mathrm{h}}$ identification 
efficiency of this algorithm is estimated to be approximately $60 \%$ for $p_{\mathrm{T}}>20 \mathrm{GeV}$, and it is measured in a sample enriched in $\mathrm{Z} \rightarrow \tau \tau \rightarrow \mu \tau_{\mathrm{h}}$ data events with a "tag-and-probe" technique [46]. The corresponding probability for generic hadronic jets to be misidentified as $\tau_{\mathrm{h}}$ is less than $1 \%$ [46].

For the $\mathrm{e} \tau_{\mathrm{h}}\left(\mu \tau_{\mathrm{h}}\right)$ final state, data are collected with a trigger requiring at least one isolated electron (muon) with a threshold of $p_{\mathrm{T}}>27(24) \mathrm{GeV}$.

Events are selected by requiring one isolated electron (muon) with transverse momentum $p_{\mathrm{T}}>30(26) \mathrm{GeV}$ and $|\eta|<2.4$, at least two jets with $p_{\mathrm{T}}>30 \mathrm{GeV}$ and $|\eta|<2.5$, and exactly one $\tau_{\mathrm{h}}$ candidate with $p_{\mathrm{T}}>30 \mathrm{GeV}$ and $|\eta|<2.4$. The $\tau_{\mathrm{h}}$ candidate and the selected lepton are required to have opposite electric charges (OC). Electrons or muons are required to be separated from any jet and from the $\tau_{\mathrm{h}}$ candidate in the $\eta-\varphi$ plane by a distance $\Delta R>0.4$. Events with any additional loosely isolated electron (muon) of $p_{\mathrm{T}}>15$ (10) $\mathrm{GeV}$ are rejected. An electron is considered loosely isolated if $I_{\text {rel }}<0.0994$ in the barrel or $I_{\text {rel }}<0.107$ in the endcaps. A muon is loosely isolated if $I_{\text {rel }}<0.25$ in either the barrel or the endcaps. At least one jet is required to be identified as originating from $b$ quark hadronization ("b tagged"). The b tagging algorithm used ("CSVv2" in ref. [47]) combines the information of displaced tracks and secondary vertices associated with the jet in a multivariate technique. The working point selected provides a $b$ tagging efficiency of about $66 \%$ with a corresponding light-flavour misidentification rate of $1 \%$. The selected events exhibit good agreement between the observed data and the expectation, as shown in figure 1 for the $p_{\mathrm{T}}$ distribution of the $\tau_{\mathrm{h}}$ candidate. The dominant background contribution comes from other $t \bar{t}$ decays, mostly from lepton+jets final states where a jet is misidentified as a $\tau_{\mathrm{h}}$ candidate.

\section{Event categories and fit procedure}

The $t \bar{t}$ production cross section is extracted from a PLR fit of the binned distribution of the transverse mass of the lepton and $p_{\mathrm{T}}^{\text {miss }}$ in two kinematic event categories, for each of the $\mathrm{e} \tau_{\mathrm{h}}$

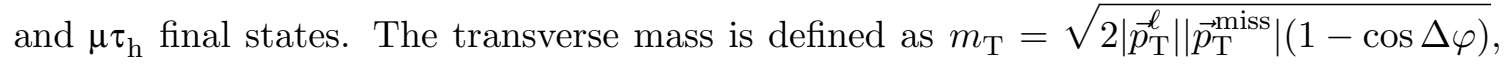
where $\Delta \varphi$ is the azimuthal angle difference between the lepton transverse momentum vector, $\vec{p}_{\mathrm{T}}^{\ell}$, and $\vec{p}_{\mathrm{T}}^{\text {miss }}$. The $m_{\mathrm{T}}$ distribution provides separation between signal and background processes (as shown in figure 2) and does not significantly depend on $p_{\mathrm{T}}$ and $\eta$ of the $\tau$ candidate, or other jet characteristics in the kinematic ranges of this study. Two event categories are defined according to the kinematic properties of jets in the event. In order to discriminate against the main background of misidentified $\tau_{\mathrm{h}}$ from the $t \overline{\mathrm{t}}$ lepton + jets process, the constraints from top quark and $\mathrm{W}$ boson masses in the decay $\mathrm{t} \rightarrow \mathrm{bW} \rightarrow \mathrm{b}\left(\mathrm{q} \overline{\mathrm{q}}^{\prime}\right)$ are used. Jet triplets are constructed for each combination of one b-tagged jet and two untagged jets, chosen from all jets in the event, including the $\tau_{\mathrm{h}}$ candidate. The distance parameter for each triplet is calculated as $D_{\mathrm{jjb}}=\sqrt{\left(m_{\mathrm{W}}-m_{\mathrm{jj}}\right)^{2}+\left(m_{\mathrm{t}}-m_{\mathrm{jjb}}\right)^{2}}$, where $m_{\mathrm{t}}=172.5 \mathrm{GeV}$ and $m_{\mathrm{W}}=80.385 \mathrm{GeV}$ are, respectively, the masses of the top quark and of the $\mathrm{W}$ boson [48], $m_{\mathrm{jj}}$ is the invariant mass of the two untagged jets, and $m_{\mathrm{jjb}}$ is the invariant mass of the jet triplet. The event is assigned to the "signal-like" category if there is only one untagged jet, or if the minimum parameter value $D_{\mathrm{jjb}}^{\min }$ is larger than $60 \mathrm{GeV}$. 

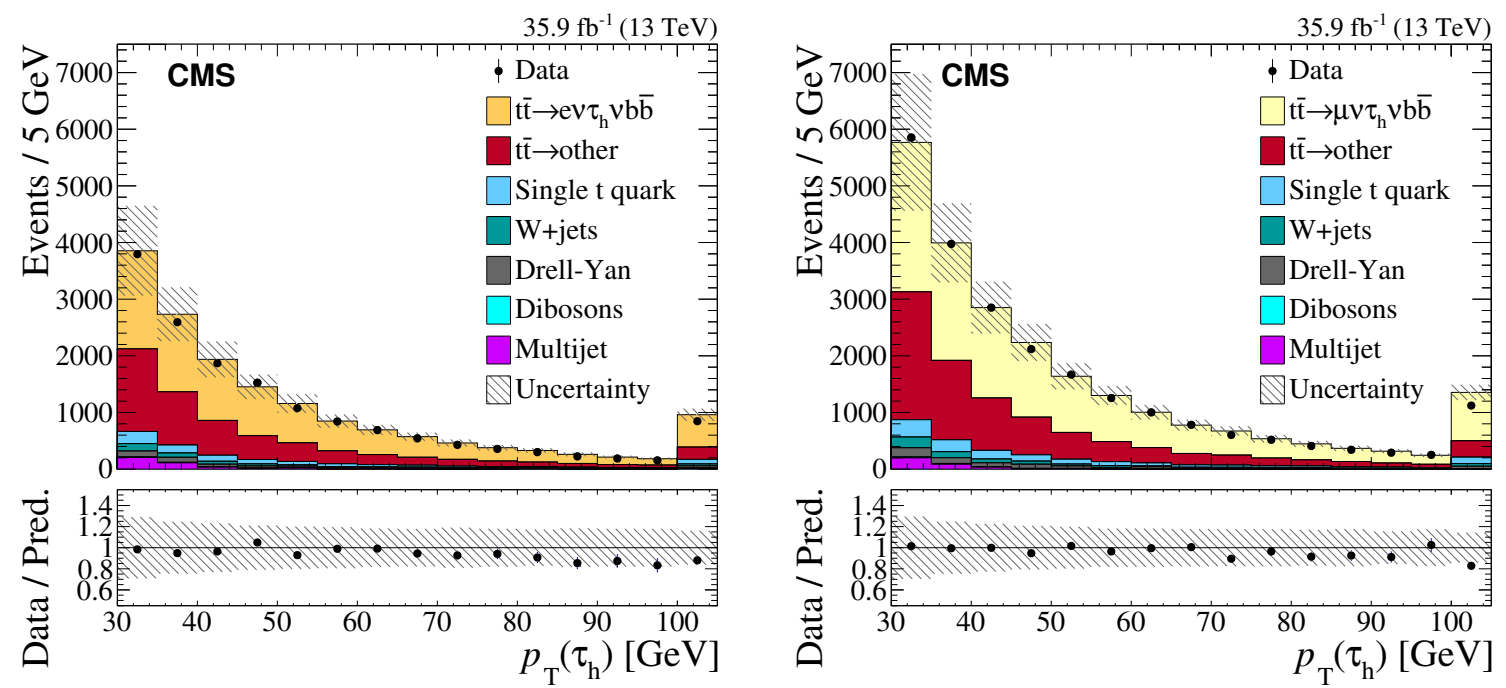

Figure 1. The $\tau_{\mathrm{h}} p_{\mathrm{T}}$ distributions for events of the $\mathrm{e} \tau_{\mathrm{h}}$ (left) and $\mu \tau_{\mathrm{h}}$ (right) final states observed prior to fitting. Distributions obtained from data (filled circles) are compared with simulation (shaded histograms). The last bin includes overflow events. The simulated contributions are normalized to the cross section values predicted in the SM. The main processes are shown: the signal, the other $t \bar{t}$ processes grouped together, single top quark production, $\mathrm{W}+$ jets, DY processes, diboson, and multijet production. The ratio of the data to the total SM prediction is shown in the lower panel. The hatched bands indicate the systematic uncertainties and the statistical uncertainties of all simulated samples. Statistical uncertainties on the data points are not visible because of the scale of the figure.

Otherwise, it is assigned to the "background-like" event category. The threshold of $60 \mathrm{GeV}$ provides an optimal separation of signal and background event categories, together with a maximization of the yields in each of the two categories in order to reduce the statistical uncertainties. In the fit, the two event categories provide an additional constraint on the background processes independent from the details of the $m_{\mathrm{T}}$ distribution.

The cross section is derived from the signal strength measured in the fit, i.e. its ratio to the value expected in the SM. It is estimated for both event categories, in each of the $\mathrm{e} \tau_{\mathrm{h}}$ and $\mu \tau_{\mathrm{h}}$ final states. The expected number of events in a given bin of the $m_{\mathrm{T}}$ distribution is parametrized as a function of signal strength and nuisance parameters. The nuisance parameters encode the effects of systematic uncertainties. The signal strength is a free parameter in the fit. The fitted variables do not significantly depend on the kinematic properties of the $\tau$ lepton in the specific t $\bar{t}$ signal model considered here, i.e. $\mathrm{t} \overline{\mathrm{t}} \rightarrow\left(\ell \nu_{\ell}\right)\left(\tau_{\mathrm{h}} \nu_{\tau}\right) \mathrm{b} \overline{\mathrm{b}}$. The likelihood function is defined as a product of Poisson distributions of the expected number of events in bins of the $m_{\mathrm{T}}$ distribution and nuisance constraints. Based on the likelihood function, the PLR test statistic is defined as the ratio between the maximum of the likelihood for a given value of signal strength and the global maximum of the likelihood function. The effect of the systematic uncertainties on the signal strength is determined with this approach. 

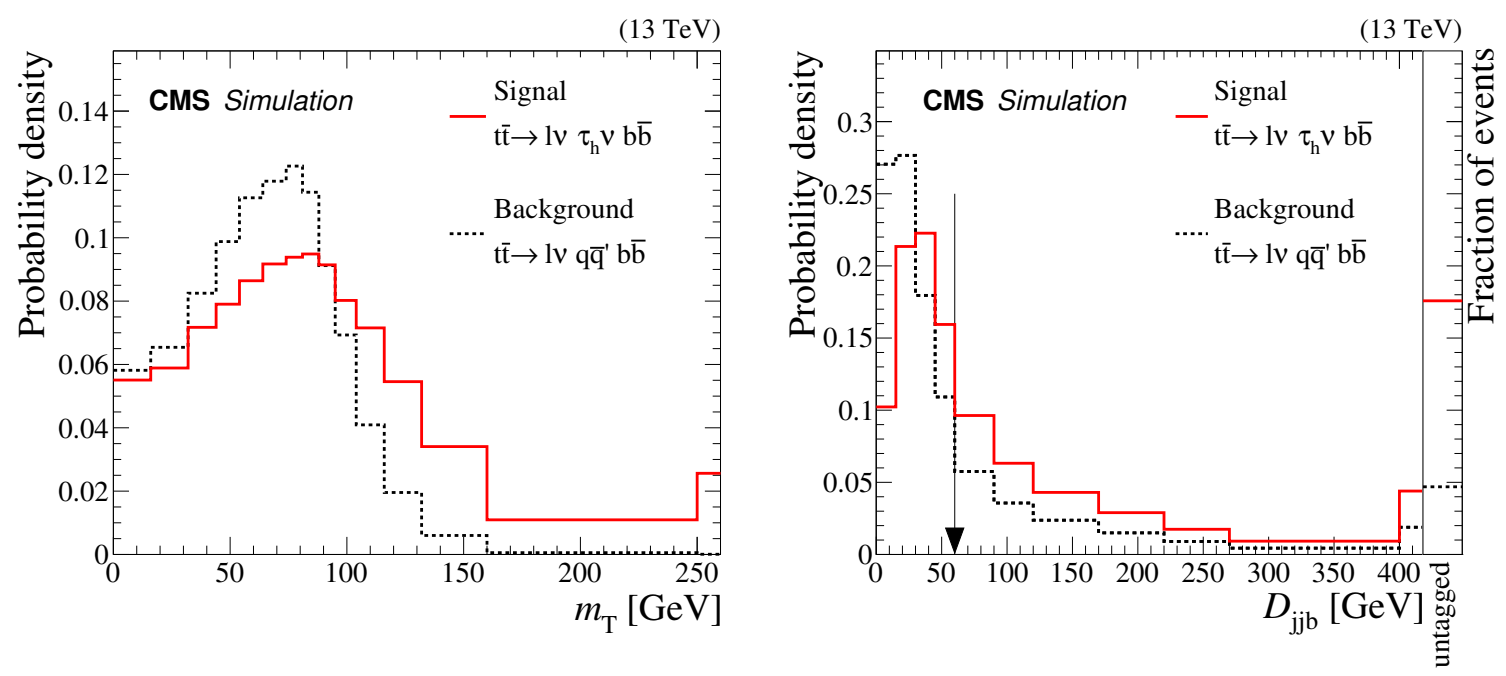

Figure 2. Comparison of the signal $\left(\mathrm{t} \overline{\mathrm{t}} \rightarrow \ell \nu_{\ell} \tau_{\mathrm{h}} \nu_{\tau} \mathrm{b} \overline{\mathrm{b}}\right)$ and the main background of misidentified $\tau_{\mathrm{h}}$ $\left(\mathrm{t} \overline{\mathrm{t}} \rightarrow \ell \nu_{\ell} \mathrm{q} \overline{\mathrm{q}}^{\prime} \mathrm{b} \overline{\mathrm{b}}\right)$ in the shapes of the normalized distributions of the transverse mass $m_{\mathrm{T}}$ between the lepton and $p_{\mathrm{T}}^{\text {miss }}$ (left), and the $D_{\mathrm{jjb}}^{\mathrm{min}}$ parameter (see text) of the event categories (right). In the $m_{\mathrm{T}}$ distribution, the signal may extend beyond the $\mathrm{W}$ boson mass endpoint because of the two-neutrino final state, whereas the background process cannot. The last bin in both distributions includes overflow events. In the $D_{\mathrm{jjb}}^{\mathrm{min}}$ distribution, the downward arrow points at the threshold of the cut used $\left(D_{\mathrm{jjb}}^{\min }>60 \mathrm{GeV}\right)$, and the panel on the right shows the fraction of events in the "signal-like" category where there is only one untagged jet, which amounts to approximately $5 \%$ of all background events and $17 \%$ of all signal events.

\section{Background estimate}

The main background contribution comes from events with one lepton, significant $p_{\mathrm{T}}^{\text {miss }}$, and three or more jets, dominated by the lepton+jets t $\bar{t}$ process, where one of the jets is falsely identified as a $\tau_{\mathrm{h}}$. Misidentified $\tau_{\mathrm{h}}$ candidates also come from multijet and $\mathrm{W}+$ jet background processes. There is a small contribution from processes with genuine hadronic $\tau_{\mathrm{h}}$ : tW single top quark production, $\tau_{\ell} \tau_{\mathrm{h}}$ from DY decays, $\mathrm{t} \overline{\mathrm{t}} \rightarrow \tau_{\ell} \tau_{\mathrm{h}} \mathrm{b} \overline{\mathrm{b}}$, and diboson processes. All processes, except multijet, are estimated from simulation after applying appropriate corrections. The pileup, trigger efficiencies, lepton identification, jet energy corrections, and b tagging efficiencies in the simulation are corrected with scale factors measured in separate publications $[43,45,49]$, as described in section 7 .

The $\tau_{\mathrm{h}}$ misidentification contribution is determined by constraining the falsely identified $\tau_{\mathrm{h}}$ in the overall fit to the data in the $m_{\mathrm{T}}$ distribution. In the fit, the event yields of the background processes with a misidentified $\tau_{\mathrm{h}}$ are determined by adjusting the normalization of the shapes of the $m_{\mathrm{T}}$ distributions. The normalization factors are introduced as nuisance parameters with constraints determined from studies in other processes [46]. The corresponding uncertainties are discussed in section 7 .

The background from the multijet processes is determined from data as it provides a more accurate description with a smaller statistical uncertainty. The shape of the $m_{\mathrm{T}}$ distribution is obtained from a sample of events containing lepton and $\tau_{\mathrm{h}}$ candidates of 
the same charge (SC). It is estimated by subtracting from the data all other processes taken from simulation, including the fully hadronic final states in t $\bar{t}$, single top quark, and dibosons. The $m_{\mathrm{T}}$ shapes for SC and $\mathrm{OC}$ events are the same within the uncertainties in a control region with a relaxed $\tau_{\mathrm{h}}$ identification requirement, and in agreement with the simulation. The normalization is corrected by multiplying the SC $m_{\mathrm{T}}$ distribution by the OC-to-SC ratio, $f_{\mathrm{OC} / \mathrm{SC}}$, as determined in a control region from events with a relaxed $\tau_{\mathrm{h}}$ identification and an inverted lepton isolation requirement, where the multijet contribution is dominant. All other event selection requirements remain the same as in the main selection. The ratio is measured to be $f_{\mathrm{OC} / \mathrm{SC}}=1.05 \pm 0.05$ (stat + syst), in agreement with simulation. As one of the processes with misidentified $\tau_{\mathrm{h}}$, the normalization of the multijet contribution is varied in the fit as a separate nuisance parameter, as described in section 7 .

\section{Systematic uncertainties}

The main sources of systematic uncertainty are from $\tau_{\mathrm{h}}$ identification and misidentification, b tagging, estimation of pileup in the pp collisions, jet energy scale (JES), and jet energy resolution (JER). Other sources of uncertainty are from lepton identification, trigger efficiency, and the calibration of the integrated luminosity. Theoretical uncertainties are also included in the event simulation. Uncertainties are applied in a coherent way to signal and background processes. The corresponding corrections and their uncertainties are measured in dedicated studies, which are described below.

The uncertainty in the efficiency of $\tau_{\mathrm{h}}$ identification is $5 \%$ for all $\tau_{\mathrm{h}}$ with $p_{\mathrm{T}}>20 \mathrm{GeV}$ and is applied to all processes with a genuine $\tau_{\mathrm{h}}$. It is measured with a tag-and-probe technique in samples enriched in $\mathrm{Z} \rightarrow \tau_{\ell} \tau_{\mathrm{h}}$ events [46]. The $\tau_{\mathrm{h}}$ charge confusion probability, estimated to be less than $1 \%$, is considered a part of the $\tau_{\mathrm{h}}$ identification efficiency uncertainty. The correction to the reconstructed energy of the $\tau_{\mathrm{h}}$ jet ( $\tau$ energy scale) and the corresponding uncertainty is estimated in a fit of the data in distributions sensitive to the $\tau$ energy, such as the $\tau_{\mathrm{h}}$ visible mass [46]. The dominant background contribution arises from processes where a jet is misidentified as $\tau_{\mathrm{h}}$, mainly lepton + jets $\mathrm{t} \overline{\mathrm{t}}, \mathrm{W}+$ jets, and multijet production. The $\tau_{\mathrm{h}}$ misidentification probability and its uncertainty in these processes are directly measured in the fit. The misidentification probability is varied within $\pm 50 \%$ of the expected values in all processes with a jet falsely identified as the $\tau_{\mathrm{h}}$ candidate. The variation covers the differences between expected and observed misidentification probabilities and the possible dependence on other kinematic properties of the $\tau_{\mathrm{h}}$ candidate [46]. The misidentification probability is significantly constrained in the fit and is not the dominant source of the uncertainty in the final result.

The uncertainties related to b tagging (mistagging) efficiencies are estimated from a variety of control samples enriched in b quarks (c and light-flavour quarks) [47]; the datato-simulation scale factors for b, c, and light-flavour jets are applied to the simulation and the corresponding uncertainties are included in the fit.

The uncertainties in the JES, JER, and $p_{\mathrm{T}}^{\text {miss }}$ scales are estimated according to the prescription described in ref. [50]. The uncertainty in the JES is evaluated as a function of jet $p_{\mathrm{T}}$ and $\eta$. The JES and JER uncertainties are propagated to the $p_{\mathrm{T}}^{\text {miss }}$ scale. 
The lepton trigger, identification, and isolation efficiencies are measured in data and simulation with a tag-and-probe method in $\mathrm{Z} \rightarrow \ell^{+} \ell^{-}$events [43-45]. The simulated events are corrected with the corresponding data-to-simulation scale factors. The uncertainties in the scale factors are included as systematic uncertainties in the measurement.

The uncertainty in the integrated luminosity is estimated to be $2.5 \%$ [51].

The pileup distribution is estimated from the measured luminosity in each bunch crossing multiplied by the average total inelastic cross section. It is used to model the pileup in simulation with an uncertainty obtained by varying the inelastic pp cross section extracted from a control region by its uncertainty of $\pm 4.6 \%$ [49].

The measurement includes the uncertainty in the modelling of the $\mathrm{b}$ quark fragmentation, which covers $\mathrm{e}^{+} \mathrm{e}^{-}$data [52-55] at the $\mathrm{Z}$ pole with the Bowler-Lund [56] and Peterson [57] parametrizations, and the uncertainties in the semileptonic b-flavoured hadron branching fractions according to their measured values [48]. An uncertainty in the modelling of the $p_{\mathrm{T}}$ distribution of the top quark in t $\overline{\mathrm{t}}$ processes is included to cover the difference between the predicted and observed spectra [58-61]. The fit is sensitive to the top quark $p_{\mathrm{T}}$ as it affects the shape of the $m_{\mathrm{T}}$ distribution. The top quark $p_{\mathrm{T}}$ variation also covers the slight trend of the $\tau_{\mathrm{h}} p_{\mathrm{T}}$ distribution.

The cross section is measured by the fit in the fiducial phase space of the detector. The fiducial cross section is extrapolated to the full phase space by correcting for the acceptance of the t $\overline{\mathrm{t}}$ signal process. The fit and the acceptance include the following modelling uncertainties: the renormalization and factorization scales, and PDFs including $\alpha_{S}$. The uncertainty in the PDF is estimated by using the CT14 (NNLO) set as alternative PDFs. The renormalization and factorization scales in the $\mathrm{ME}$ calculations are varied independently by factors of 0.5 and 2.0 from their nominal values, and the envelope of the variations is included in the measurement. The scale is varied by factors of 0.5 and 2.0 in the parton shower (PS) simulation of final-state and initial-state radiation, FSR and ISR. The $h_{\text {damp }}$ parameter regulating the real emissions in POWHEG (ME-PS matching) is varied from its central value of $1.58 m_{\mathrm{t}}$ using samples with $h_{\text {damp }}$ set to $0.99 m_{\mathrm{t}}$ and $2.24 m_{\mathrm{t}}\left(m_{\mathrm{t}}=172.5 \mathrm{GeV}\right)$, as obtained from tuning this parameter to $\mathrm{t} \overline{\mathrm{t}}$ data at $\sqrt{s}=8 \mathrm{TeV}$ [62]. The underlying event tune is varied within its uncertainties [23,62]. The effect of these uncertainties on the final state objects is included in the fit in the fiducial phase space by adding the corresponding systematic variations normalized to the nominal acceptance. Therefore, the measurement in the fiducial phase space is performed with the nominal acceptance and its uncertainties are only included in the extrapolation to the full phase space. The uncertainties in the fit are not correlated with the acceptance uncertainty in the extrapolation to the full phase space.

The theoretical uncertainties are implemented by reweighting the simulated events with corresponding scale factors. The differences between weighted and unweighted distributions are taken as the uncertainties in the modelling. Separate data sets with varied parameters are used for determining FSR, ISR, ME-PS matching, and underlying event uncertainties.

The impact of the systematic uncertainties on the measurement is given in table 2 . 


\begin{tabular}{|ccccc|}
\hline \multicolumn{4}{c}{ e $\tau_{\mathrm{h}}$} & \multicolumn{2}{c|}{$\mu \tau_{\mathrm{h}}$} \\
\multicolumn{1}{c}{ Process } & Background-like & Signal-like & Background-like & Signal-like \\
\hline $\begin{array}{c}\text { Signal } \\
\mathrm{t} \overline{\mathrm{t}} \rightarrow\left(\ell \nu_{\ell}\right)\left(\tau_{\mathrm{h}} \nu_{\tau}\right) \mathrm{b} \overline{\mathrm{b}}\end{array}$ & $3440 \pm 40 \pm 210$ & $5320 \pm 40 \pm 360$ & $5140 \pm 40 \pm 130$ & $7890 \pm 50 \pm 280$ \\
$\mathrm{t} \overline{\mathrm{t}}$ backgrounds & & & \\
$\mathrm{t} \overline{\mathrm{t}} \rightarrow\left(\ell \nu_{\ell}\right)\left(\mathrm{q} \overline{\mathrm{q}}^{\prime}\right) \mathrm{b} \overline{\mathrm{b}}$ & $2450 \pm 30 \pm 1210$ & $1610 \pm 20 \pm 830$ & $3670 \pm 40 \pm 1810$ & $2440 \pm 30 \pm 1260$ \\
$\mathrm{t} \overline{\mathrm{t}} \rightarrow$ other & $390 \pm 10 \pm 70$ & $510 \pm 10 \pm 80$ & $580 \pm 10 \pm 110$ & $760 \pm 20 \pm 120$ \\
Other backgrounds & & & & \\
Single t quark & $370 \pm 10 \pm 90$ & $540 \pm 10 \pm 100$ & $500 \pm 10 \pm 110$ & $790 \pm 10 \pm 150$ \\
Drell-Yan & $150 \pm 20 \pm 20$ & $310 \pm 20 \pm 20$ & $200 \pm 20 \pm 10$ & $410 \pm 30 \pm 40$ \\
Total & $7090 \pm 80 \pm 1230$ & $8930 \pm 80 \pm 920$ & $10490 \pm 90 \pm 1820$ & $12970 \pm 90 \pm 1310$ \\
Data & 6787 & 8633 & 9931 & 13085 \\
\hline
\end{tabular}

Table 1. Expected and observed event yields in the $\ell \tau_{\mathrm{h}}(\ell=\mathrm{e}, \mu)$ final state for signal and background processes for an integrated luminosity of $35.9 \mathrm{fb}^{-1}$. Statistical and systematic uncertainties are shown. The expected prefit contributions of all processes are presented separately for background-like and signal-like event categories. The statistical uncertainties of the modelling are shown for the processes estimated from the simulation. The multijet contribution and the corresponding statistical uncertainties are estimated using data, as described in section 6 .

\section{Results}

The event yields expected from the signal and background processes, as well as the observed event yields are summarized in table 1, for the signal-like and the background-like event categories (described in section 5) in each of the $\mathrm{e} \tau_{\mathrm{h}}$ and $\mu \tau_{\mathrm{h}}$ final states. The observed event yields in data show good agreement with the prediction. The $m_{\mathrm{T}}$ distributions in the two categories of the selected events are shown in figure 3 , for both the e $\tau_{\mathrm{h}}$ and $\mu \tau_{\mathrm{h}}$ final states. A good shape agreement is observed between the data and the expected sum of signal and background distributions.

Table 2 lists the systematic uncertainties in the signal strength after the fit. The effect of the uncertainties on the signal strength is estimated by a likelihood scan where only one nuisance parameter (or a group of them) is varied at once while the others are fixed to their nominal postfit values. The largest experimental uncertainties are from $\tau_{\mathrm{h}}$ identification and misidentification, and pileup estimation. The largest theoretical uncertainties are due to the modelling of top quark $p_{\mathrm{T}}$ in $\mathrm{t} \overline{\mathrm{t}}$ processes, b quark fragmentation, and PS modelling (ISR and FSR). 

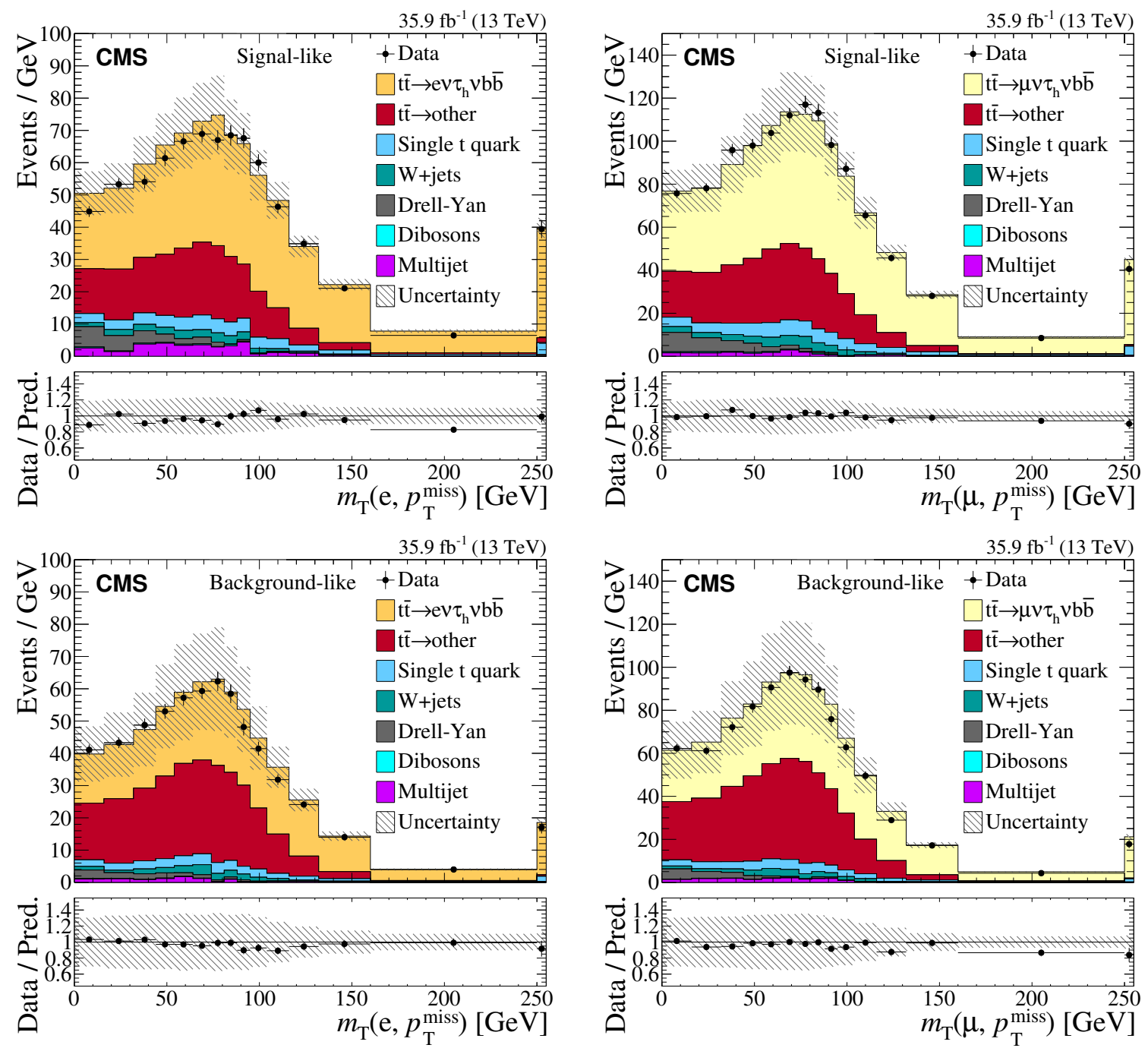

Figure 3. The transverse mass distributions between lepton (e or $\mu$ ) and $p_{\mathrm{T}}^{\text {miss }}, m_{\mathrm{T}}$, in the signallike (upper) and background-like (lower) event categories for the $\mathrm{e} \tau_{\mathrm{h}}$ (left) and $\mu \tau_{\mathrm{h}}$ (right) final states observed prior to fitting. Distributions obtained from data (filled circles) are compared with simulation (shaded histograms). The last bin includes overflow events. The simulated contributions are normalized to the cross section values predicted in the SM. The main processes are shown: the signal, the other $t \bar{t}$ processes grouped together, single top quark production, $\mathrm{W}+$ jets, DY processes, diboson, and multijet production. The ratio of the data to the total SM prediction is shown in the lower panel. The vertical bars on the data points indicate the statistical uncertainties, the hatched band indicates the systematic uncertainties and the statistical uncertainties in all simulated samples. 


\begin{tabular}{|c|c|c|c|c|c|}
\hline \multirow[t]{2}{*}{ Source } & \multicolumn{5}{|c|}{ Uncertainty [\%] } \\
\hline & $\mathrm{e} \tau_{\mathrm{h}}$ & $\mu \tau_{\mathrm{h}}$ & Combined & Dileptons & Correlation \\
\hline \multicolumn{6}{|l|}{ Experimental uncertainties } \\
\hline$\tau_{\mathrm{h}}$ jet identification & 4.7 & 4.5 & 4.5 & - & 0 \\
\hline$\tau_{\mathrm{h}}$ jet misidentification & 2.2 & 2.3 & 2.3 & - & 0 \\
\hline Pileup & 2.5 & 2.2 & 2.3 & 0.1 & 1 \\
\hline Lepton identification and isolation & 1.8 & 1.1 & 1.2 & 2.0 & 1 \\
\hline b tagging efficiency & 1.1 & 1.2 & 0.9 & 0.4 & 1 \\
\hline$\tau_{\mathrm{h}}$ energy scale & 0.7 & 0.8 & 0.8 & - & 0 \\
\hline Trigger efficiency & 2.3 & 0.6 & 0.7 & 0.3 & 0 \\
\hline Drell-Yan background & 0.4 & 0.4 & 0.6 & 0.9 & 1 \\
\hline $\mathrm{t} \overline{\mathrm{t}}$ background & 1.0 & 0.8 & 0.6 & 0.2 & 0 \\
\hline tW background & 0.6 & 0.5 & 0.5 & 1.1 & 1 \\
\hline $\mathrm{W}+$ jets background & 0.1 & 0.4 & 0.5 & 0.2 & 0 \\
\hline Multijet background & 0.1 & 0.5 & 0.4 & $<0.1$ & 0 \\
\hline Jet energy scale & 0.1 & 0.2 & 0.4 & 0.4 & 1 \\
\hline Jet energy resolution & 0.6 & 0.3 & 0.1 & 0.4 & 1 \\
\hline Electron momentum scale & 0.1 & 0.1 & 0.1 & 0.1 & 1 \\
\hline Muon momentum scale & 0.1 & 0.1 & 0.1 & 0.1 & 1 \\
\hline Diboson background & $<0.1$ & $<0.1$ & $<0.1$ & 0.2 & 1 \\
\hline \multicolumn{6}{|l|}{ Theoretical uncertainties } \\
\hline b fragmentation & 2.3 & 2.0 & 2.4 & 0.7 & 1 \\
\hline Top quark $p_{\mathrm{T}}$ modelling & 2.7 & 2.3 & 2.2 & 0.5 & 1 \\
\hline 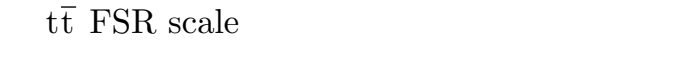 & 1.7 & 1.9 & 1.7 & 0.8 & 1 \\
\hline tW FSR scale & $<0.1$ & $<0.1$ & $<0.1$ & 0.1 & 1 \\
\hline tĒ ISR scale & 1.7 & 1.6 & 1.5 & 0.4 & 1 \\
\hline tW ISR scale & $<0.1$ & $<0.1$ & $<0.1$ & 0.1 & 1 \\
\hline $\mathrm{t} \overline{\mathrm{t}} \mathrm{ME}$ scale & 1.1 & 1.2 & 1.1 & 0.2 & 1 \\
\hline tW ME scale & $<0.1$ & $<0.1$ & $<0.1$ & 0.2 & 1 \\
\hline Drell-Yan ME scale & $<0.1$ & $<0.1$ & $<0.1$ & 0.1 & 1 \\
\hline Semileptonic b hadron branching fraction & 0.8 & 0.6 & 0.7 & 0.1 & 1 \\
\hline Underlying event & 0.5 & 0.5 & 0.6 & 0.3 & 1 \\
\hline ME-PS matching & 0.4 & 0.4 & 0.5 & 0.2 & 1 \\
\hline Colour reconnection & $<0.1$ & $<0.1$ & $<0.1$ & 0.3 & 1 \\
\hline PDFs & 1.5 & 1.5 & 1.6 & 1.1 & 1 \\
\hline \multicolumn{6}{|l|}{ Normalization uncertainties } \\
\hline Statistical & 1.4 & 1.1 & 0.9 & 0.2 & 0 \\
\hline MC statistical & 2.0 & 1.6 & 1.6 & 1.1 & 0 \\
\hline Integrated luminosity & 2.5 & 2.5 & 2.5 & 2.5 & 1 \\
\hline
\end{tabular}




\begin{tabular}{|c|c|c|c|c|c|}
\hline \multirow[t]{2}{*}{ Source } & \multicolumn{5}{|c|}{ Uncertainty [\%] } \\
\hline & $\mathrm{e} \tau_{\mathrm{h}}$ & $\mu \tau_{\mathrm{h}}$ & Combined & Dileptons & Correlation \\
\hline \multicolumn{6}{|l|}{ Extrapolation uncertainties } \\
\hline $\mathrm{t} \overline{\mathrm{t}} \mathrm{ME}$ scale & 0.3 & 0.4 & 0.3 & 0.3 & 0 \\
\hline PDFs & 1.2 & 1.4 & 1.3 & 1.0 & 0 \\
\hline Top quark $p_{\mathrm{T}}$ modelling & 1.0 & 1.1 & 1.1 & 0.5 & 0 \\
\hline tE ISR scale & 0.5 & 0.3 & 0.3 & 0.1 & 0 \\
\hline tĒ FSR scale & 1.9 & 2.0 & 1.9 & 0.1 & 0 \\
\hline Underlying event & 0.3 & 0.2 & 0.2 & $<0.1$ & 0 \\
\hline
\end{tabular}

Table 2. Systematic and statistical uncertainties determined from the fit to the data in the $\mathrm{e} \tau_{\mathrm{h}}$ and $\mu \tau_{\mathrm{h}}$ final states, and their combination. Uncertainties are grouped by their origin: experimental, theoretical, normalization, and extrapolation. The uncertainties in the measurement in the dilepton final state [14] used in the partial width ratio estimate are also quoted (column "dileptons"), where the asymmetric extrapolation uncertainties are symmetrized by adding them in quadrature. As both measurements use the same data, some uncertainties in the $\ell \tau_{\mathrm{h}}$ and light dilepton final states are correlated, as shown in the last column.

The fiducial cross section for the production of $t \bar{t}$ events is extracted from the acceptance region of kinematic phase space defined by the selection criteria described earlier. The estimate of the fiducial cross section includes the branching fractions of the final states, trigger, lepton identification and isolation, and the overall reconstruction efficiency. The cross sections in the fiducial phase space for the individual $\mathrm{e} \tau_{\mathrm{h}}$ and $\mu \tau_{\mathrm{h}}$ final states, as well as the $\ell \tau_{\mathrm{h}}$ combined final state, are measured from the PLR fit to be:

$$
\begin{aligned}
& \sigma_{\mathrm{t} \overline{\mathrm{t}}}^{\mathrm{fid}}\left(\mathrm{e} \tau_{\mathrm{h}}\right)=133.2 \pm 1.9(\text { stat }) \pm 10.9(\text { syst }) \pm 3.3(\text { lumi }) \mathrm{pb} \\
& \sigma_{\mathrm{t} \overline{\mathrm{t}}}^{\mathrm{fid}}\left(\mu \tau_{\mathrm{h}}\right)=135.2 \pm 1.5(\text { stat }) \pm 9.9(\text { syst }) \pm 3.4(\text { lumi }) \mathrm{pb} \\
& \sigma_{\mathrm{t} \mathrm{t}}^{\text {fid }}\left(\ell \tau_{\mathrm{h}}\right)=134.5 \pm 1.2(\text { stat }) \pm 9.5(\text { syst }) \pm 3.4(\text { lumi }) \mathrm{pb}
\end{aligned}
$$

The acceptance $A_{\mathrm{t} \overline{\mathrm{t}}}$ is the fraction of signal events in the fiducial phase space, and it is determined with respect to all signal events in the nominal $t \bar{t}$ simulation. It includes kinematic selection cuts and is evaluated for the different signal final states as:

$$
\begin{aligned}
& A_{\mathrm{t} \overline{\mathrm{t}}}\left(\mathrm{e} \tau_{\mathrm{h}}\right)=0.1687 \pm 0.0004(\text { stat }) \pm 0.0060 \text { (syst) } \\
& A_{\mathrm{t} \overline{\mathrm{t}}}\left(\mu \tau_{\mathrm{h}}\right)=0.1756 \pm 0.0004(\text { stat }) \pm 0.0065 \text { (syst), } \\
& A_{\mathrm{t} \overline{\mathrm{t}}}\left(\ell \tau_{\mathrm{h}}\right)=0.1722 \pm 0.0003 \text { (stat) } \pm 0.0062 \text { (syst), }
\end{aligned}
$$

where the systematic uncertainties include the uncertainties of the modelling as described in section 7 and listed as "Extrapolation uncertainties" in table 2. 
The cross section values in the full phase space are obtained from the extrapolation of the fiducial cross sections using the acceptances $A_{\mathrm{t} \overline{\mathrm{t}}}$ estimated from the simulation:

$$
\begin{aligned}
& \sigma_{\mathrm{t} \overline{\mathrm{t}}}\left(\mathrm{e} \tau_{\mathrm{h}}\right)=789 \pm 11 \text { (stat) } \pm 71 \text { (syst) } \pm 20(\text { lumi }) \mathrm{pb}, \\
& \sigma_{\mathrm{t} \overline{\mathrm{t}}}\left(\mu \tau_{\mathrm{h}}\right)=770 \pm 8(\text { stat }) \pm 63(\text { syst }) \pm 20(\text { lumi }) \mathrm{pb} \\
& \sigma_{\mathrm{t} \overline{\mathrm{t}}}\left(\ell \tau_{\mathrm{h}}\right)=781 \pm 7(\text { stat }) \pm 62(\text { syst }) \pm 20 \text { (lumi) pb. }
\end{aligned}
$$

The expected and observed dependence of the likelihood on the cross section in the full phase space in the $\ell \tau_{\mathrm{h}}$ combined final state are shown in figure 4 . The result of the fit is consistent with the predicted SM t $\overline{\mathrm{t}}$ production cross section of $832_{-29}^{+20}$ (scale) \pm $35\left(\mathrm{PDF}+\alpha_{S}\right) \mathrm{pb}[32]$. Using simulated $\mathrm{t} \overline{\mathrm{t}}$ samples with different $m_{\mathrm{t}}$ values, we find that the cross section changes by $1.5 \%$ per $\Delta m_{\mathrm{t}}=1 \mathrm{GeV}$.

The ratio of the cross section in the $\ell \tau_{\mathrm{h}}$ final state divided by the cross section measured in the dilepton final state in the same data-taking period [14] yields a value of $R_{\ell \tau_{\mathrm{h}} / \ell \ell}=$ $0.973 \pm 0.009$ (stat) \pm 0.066 (syst), consistent with unity as expected from lepton flavour universality. The relative systematic uncertainty in the ratio is $6.8 \%$. About $5 \%$ comes from the uncertainties in the $\tau_{\mathrm{h}}$ identification (4.5\%) and misidentification probability in $t \bar{t}$ events $(2.3 \%)$. The rest comes from the other uncorrelated uncertainties in the ratio and the treatment of the correlated uncertainties in the calculation of the ratio. In particular, a small contribution comes from the uncertainties in the extrapolation to the full phase space that are considered uncorrelated because the two measurements extrapolate from different fiducial phase spaces. Also, the triggers are not the same.

The measurement also provides an estimate of the ratio of the partial to the total width of the top quark decay, $R_{\Gamma}=\Gamma\left(\mathrm{t} \rightarrow \tau \nu_{\tau} \mathrm{b}\right) / \Gamma_{\text {total }}$. The ratio is calculated as $R_{\Gamma}=\sigma_{\mathrm{t} \overline{\mathrm{t}}}\left(\ell \tau_{\mathrm{h}}\right) \mathcal{B}\left(\mathrm{W} \rightarrow \tau \nu_{\tau}\right) / \sigma_{\mathrm{t} \overline{\mathrm{t}}}(\ell \ell)$, where the cross section measured in the $\ell \tau_{\mathrm{h}}$ final state is multiplied by the branching fraction $\mathcal{B}\left(\mathrm{W} \rightarrow \tau \nu_{\tau}\right)$ and divided by the inclusive $t \overline{\mathrm{t}}$ cross section measured in the dilepton final state [14]. The $\mathrm{W}$ boson branching fraction $\mathcal{B}\left(\mathrm{W} \rightarrow \tau \nu_{\tau}\right)$ that is included in the signal acceptance is cancelled out in the multiplication. Since both measurements are performed in the same data-taking period with the same reconstruction algorithms, the uncertainty in the ratio includes the correlations between common sources of uncertainties as indicated in table 2. The estimate yields the value $R_{\Gamma}=0.1050 \pm 0.0009$ (stat) \pm 0.0071 (syst), improving over the previous measurements $[48,63,64]$. The result is dominated by the systematic uncertainty and it is consistent with the SM value of $0.1083 \pm 0.0002$ [48]. While in ref. [63] the partial width is evaluated for hadronic decays of $\tau$ leptons, here $R_{\Gamma}$ is measured for all $\tau$ decays by using the $\mathcal{B}\left(\tau \rightarrow \tau_{\mathrm{h}} v_{\tau}\right)=64.8 \pm 0.1 \%$ branching fraction [48].

\section{Summary}

A measurement of the top quark pair production cross section in the $t \bar{t} \rightarrow\left(\ell v_{\ell}\right)\left(\tau_{h} v_{\tau}\right) b \bar{b}$ channel, where $\ell$ is either an electron or a muon, is performed by CMS in proton-proton collisions at LHC, using a data sample corresponding to an integrated luminosity of $35.9 \mathrm{fb}^{-1}$ obtained at $\sqrt{s}=13 \mathrm{TeV}$. Events are selected by requiring the presence of an electron 


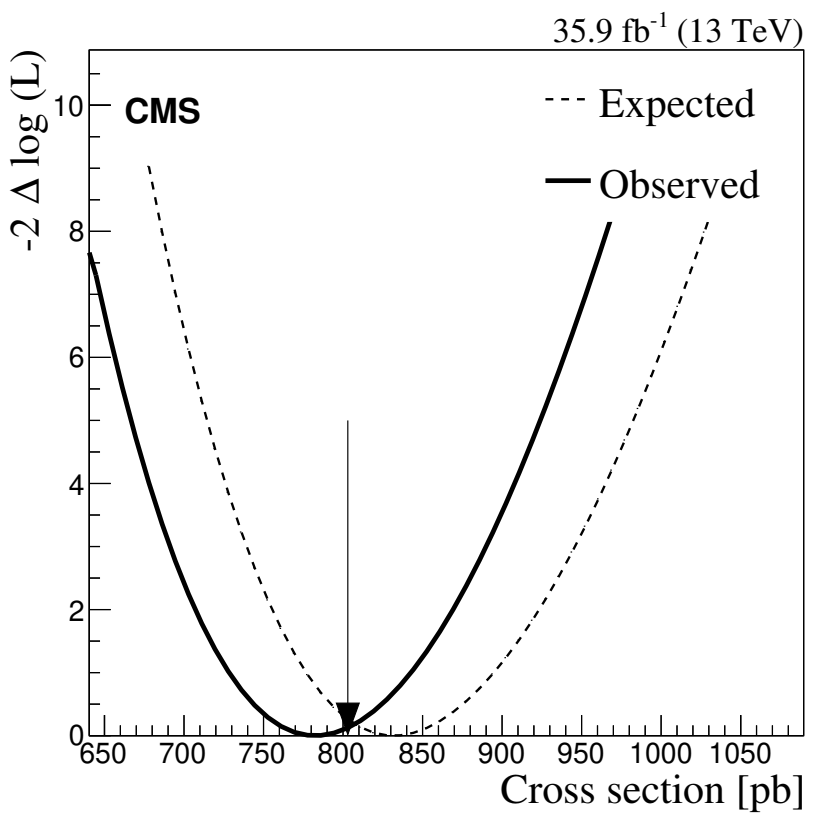

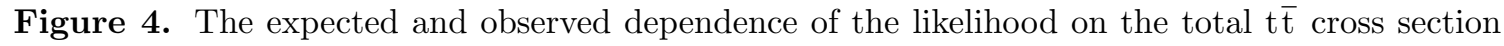
$\sigma_{\mathrm{t} \overline{\mathrm{t}}}$. It is derived from the fiducial phase space by a simple extrapolation. The arrow points at the cross section measured in the light dilepton final state. The goodness of the fit determined with a Kolmogorov-Smirnov method yields a $p$ value of 0.24 .

or a muon, and at least three jets, of which at least one is b tagged and one is identified as a $\tau$ lepton decaying to hadrons $\left(\tau_{\mathrm{h}}\right)$. The largest background contribution arises from $t \bar{t}$ lepton+jets events, $t \bar{t} \rightarrow\left(\ell v_{\ell}\right)\left(q \bar{q}^{\prime}\right) b \bar{b}$, where one jet is misidentified as the $\tau_{\mathrm{h}}$. The background contribution is constrained in a fit to the distribution of the transverse mass of the light lepton and missing transverse momentum system in two event categories, constructed according to the kinematic properties of the jets in the t $\bar{t}$ lepton+jets final state. The signal enters as a free parameter without constraining the kinematic properties of the $\tau$ lepton. Assuming a top quark mass of $172.5 \mathrm{GeV}$, the measured total $\mathrm{t} \overline{\mathrm{t}}$ cross section $\sigma_{\mathrm{t} \overline{\mathrm{t}}}\left(\ell \tau_{\mathrm{h}}\right)=781 \pm 7$ (stat) \pm 62 (syst) \pm 20 (lumi) pb is in agreement with the standard model expectation. This is the first measurement of the $t \bar{t}$ production cross section in proton-proton collisions at $\sqrt{s}=13 \mathrm{TeV}$ that explicitly includes hadronically decaying $\tau$ leptons, and it improves the relative precision with respect to the 7 and $8 \mathrm{TeV}$ results $[65,66]$. The higher precision is achieved through a shape fit to the kinematic distributions of the events, thus better constraining the backgrounds. The measurement of the ratio of the cross section in the $\ell \tau_{\mathrm{h}}$ final state to the light dilepton cross section [14] yields a value of $R_{\ell \tau_{\mathrm{h}} / \ell \ell}=0.973 \pm 0.009$ (stat) \pm 0.066 (syst), consistent with lepton universality. The ratio of the partial to the total width of the top quark $\Gamma\left(\mathrm{t} \rightarrow \tau \mathrm{v}_{\tau} \mathrm{b}\right) / \Gamma_{\text {total }}=0.1050 \pm 0.0009$ (stat) \pm 0.0071 (syst) is measured with respect to the $t \bar{t}$ inclusive cross section extrapolated from the light dilepton final state, improving the precision over the previous measurements [63, 64]. 


\section{Acknowledgments}

We congratulate our colleagues in the CERN accelerator departments for the excellent performance of the LHC and thank the technical and administrative staffs at CERN and at other CMS institutes for their contributions to the success of the CMS effort. In addition, we gratefully acknowledge the computing centres and personnel of the Worldwide LHC Computing Grid for delivering so effectively the computing infrastructure essential to our analyses. Finally, we acknowledge the enduring support for the construction and operation of the LHC and the CMS detector provided by the following funding agencies: BMBWF and FWF (Austria); FNRS and FWO (Belgium); CNPq, CAPES, FAPERJ, FAPERGS, and FAPESP (Brazil); MES (Bulgaria); CERN; CAS, MoST, and NSFC (China); COLCIENCIAS (Colombia); MSES and CSF (Croatia); RPF (Cyprus); SENESCYT (Ecuador); MoER, ERC IUT, PUT and ERDF (Estonia); Academy of Finland, MEC, and HIP (Finland); CEA and CNRS/IN2P3 (France); BMBF, DFG, and HGF (Germany); GSRT (Greece); NKFIA (Hungary); DAE and DST (India); IPM (Iran); SFI (Ireland); INFN (Italy); MSIP and NRF (Republic of Korea); MES (Latvia); LAS (Lithuania); MOE and UM (Malaysia); BUAP, CINVESTAV, CONACYT, LNS, SEP, and UASLP-FAI (Mexico); MOS (Montenegro); MBIE (New Zealand); PAEC (Pakistan); MSHE and NSC (Poland); FCT (Portugal); JINR (Dubna); MON, RosAtom, RAS, RFBR, and NRC KI (Russia); MESTD (Serbia); SEIDI, CPAN, PCTI, and FEDER (Spain); MOSTR (Sri Lanka); Swiss Funding Agencies (Switzerland); MST (Taipei); ThEPCenter, IPST, STAR, and NSTDA (Thailand); TUBITAK and TAEK (Turkey); NASU (Ukraine); STFC (United Kingdom); DOE and NSF (U.S.A.).

Individuals have received support from the Marie-Curie programme and the European Research Council and Horizon 2020 Grant, contract Nos. 675440, 752730, and 765710 (European Union); the Leventis Foundation; the A.P. Sloan Foundation; the Alexander von Humboldt Foundation; the Belgian Federal Science Policy Office; the Fonds pour la Formation à la Recherche dans l'Industrie et dans l'Agriculture (FRIA-Belgium); the Agentschap voor Innovatie door Wetenschap en Technologie (IWT-Belgium); the F.R.S.-FNRS and FWO (Belgium) under the "Excellence of Science - EOS" - be.h project n. 30820817; the Beijing Municipal Science \& Technology Commission, No. Z181100004218003; the Ministry of Education, Youth and Sports (MEYS) of the Czech Republic; the Deutsche Forschungsgemeinschaft (DFG) under Germany's Excellence Strategy — EXC 2121 "Quantum Universe" - 390833306; the Lendület ("Momentum") Programme and the János Bolyai Research Scholarship of the Hungarian Academy of Sciences, the New National Excellence Program ÚNKP, the NKFIA research grants 123842, 123959, 124845, 124850, 125105, 128713, 128786, and 129058 (Hungary); the Council of Science and Industrial Research, India; the HOMING PLUS programme of the Foundation for Polish Science, cofinanced from European Union, Regional Development Fund, the Mobility Plus programme of the Ministry of Science and Higher Education, the National Science Center (Poland), contracts Harmonia 2014/14/M/ST2/00428, Opus 2014/13/B/ST2/02543, 2014/15/B/ST2/03998, and 2015/19/B/ST2/02861, Sonata-bis 2012/07/E/ST2/01406; the National Priorities Research Program by Qatar National Research Fund; the Ministry of Science and Education, grant no. 3.2989.2017 (Russia); the Programa Estatal de Fomento de la Investigación 
Científica y Técnica de Excelencia María de Maeztu, grant MDM-2015-0509 and the Programa Severo Ochoa del Principado de Asturias; the Thalis and Aristeia programmes cofinanced by EU-ESF and the Greek NSRF; the Rachadapisek Sompot Fund for Postdoctoral Fellowship, Chulalongkorn University and the Chulalongkorn Academic into Its 2nd Century Project Advancement Project (Thailand); the Nvidia Corporation; the Welch Foundation, contract C-1845; and the Weston Havens Foundation (U.S.A.).

Open Access. This article is distributed under the terms of the Creative Commons Attribution License (CC-BY 4.0), which permits any use, distribution and reproduction in any medium, provided the original author(s) and source are credited.

\section{References}

[1] LHCb collaboration, Test of lepton flavor universality by the measurement of the $B^{0} \rightarrow D^{*-} \tau^{+} \nu_{\tau}$ branching fraction using three-prong $\tau$ decays, Phys. Rev. D 97 (2018) 072013 [arXiv: 1711.02505] [INSPIRE].

[2] LHCb collaboration, Search for lepton-universality violation in $B^{+} \rightarrow K^{+} \ell^{+} \ell^{-}$decays, Phys. Rev. Lett. 122 (2019) 191801 [arXiv:1903.09252] [INSPIRE].

[3] BaBAr collaboration, Evidence for an excess of $\bar{B} \rightarrow D^{(*)} \tau^{-} \bar{\nu}_{\tau}$ decays, Phys. Rev. Lett. 109 (2012) 101802 [arXiv: 1205.5442] [INSPIRE].

[4] BABAR collaboration, Measurement of an excess of $\bar{B} \rightarrow D^{(*)} \tau^{-} \bar{\nu}_{\tau}$ decays and implications for charged Higgs bosons, Phys. Rev. D 88 (2013) 072012 [arXiv:1303.0571] [INSPIRE].

[5] S. Bifani, S. Descotes-Genon, A. Romero Vidal and M.-H. Schune, Review of lepton universality tests in B decays, J. Phys. G 46 (2019) 023001 [arXiv:1809.06229] [InSPIRE].

[6] BeLLe collaboration, Measurement of the branching ratio of $\bar{B} \rightarrow D^{(*)} \tau^{-} \bar{\nu}_{\tau}$ relative to $\bar{B} \rightarrow D^{(*)} \ell^{-} \bar{\nu}_{\ell}$ decays with hadronic tagging at Belle, Phys. Rev. D 92 (2015) 072014 [arXiv: 1507.03233] [INSPIRE].

[7] BELLE collaboration, Measurement of the branching ratio of $\bar{B}^{0} \rightarrow D^{*+} \tau^{-} \bar{\nu}_{\tau}$ relative to $\bar{B}^{0} \rightarrow D^{*+} \ell^{-} \bar{\nu}_{\ell}$ decays with a semileptonic tagging method, Phys. Rev. D 94 (2016) 072007 [arXiv: 1607.07923] [INSPIRE].

[8] BeLle collaboration, Measurement of the $\tau$ lepton polarization and $R\left(D^{*}\right)$ in the decay $\bar{B} \rightarrow D^{*} \tau^{-} \bar{\nu}_{\tau}$, Phys. Rev. Lett. 118 (2017) 211801 [arXiv: 1612. 00529] [InSPIRE].

[9] A. Djouadi, The anatomy of electro-weak symmetry breaking. II. The Higgs bosons in the minimal supersymmetric model, Phys. Rept. 459 (2008) 1 [hep-ph/0503173] [INSPIRE].

[10] G.C. Branco, P.M. Ferreira, L. Lavoura, M.N. Rebelo, M. Sher and J.P. Silva, Theory and phenomenology of two-Higgs-doublet models, Phys. Rept. 516 (2012) 1 [arXiv:1106.0034] [INSPIRE].

[11] CMS collaboration, Search for charged Higgs bosons in the $H^{ \pm} \rightarrow \tau^{ \pm} \nu_{\tau}$ decay channel in proton-proton collisions at $\sqrt{s}=13 \mathrm{TeV}$, JHEP 07 (2019) 142 [arXiv: 1903.04560] [INSPIRE].

[12] ATLAS collaboration, Search for charged Higgs bosons decaying via $H^{ \pm} \rightarrow \tau^{ \pm} \nu_{\tau}$ in the $\tau+$ jets and $\tau+$ lepton final states with $36 \mathrm{fb}^{-1}$ of pp collision data recorded at $\sqrt{s}=13 \mathrm{TeV}$ with the ATLAS experiment, JHEP 09 (2018) 139 [arXiv:1807.07915] [INSPIRE]. 
[13] G. Cowan, K. Cranmer, E. Gross and O. Vitells, Asymptotic formulae for likelihood-based tests of new physics, Eur. Phys. J. C 71 (2011) 1554 [Erratum ibid. C 73 (2013) 2501] [arXiv: 1007.1727] [INSPIRE].

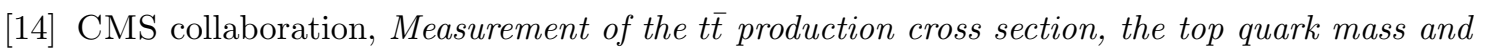
the strong coupling constant using dilepton events in pp collisions at $\sqrt{s}=13 \mathrm{TeV}$, Eur. Phys. J. C 79 (2019) 368 [arXiv:1812.10505] [INSPIRE].

[15] CMS collaboration, The CMS trigger system, 2017 JINST 12 P01020 [arXiv:1609.02366] [INSPIRE].

[16] CMS collaboration, The CMS experiment at the CERN LHC, 2008 JINST 3 S08004 [INSPIRE].

[17] P. Nason, A new method for combining NLO QCD with shower Monte Carlo algorithms, JHEP 11 (2004) 040 [hep-ph/0409146] [INSPIRE].

[18] S. Frixione, P. Nason and C. Oleari, Matching NLO QCD computations with parton shower simulations: the POWHEG method, JHEP 11 (2007) 070 [arXiv:0709.2092] [INSPIRE].

[19] S. Alioli, P. Nason, C. Oleari and E. Re, A general framework for implementing NLO calculations in shower Monte Carlo programs: the POWHEG BOX, JHEP 06 (2010) 043 [arXiv: 1002.2581] [INSPIRE].

[20] J.M. Campbell, R.K. Ellis, P. Nason and E. Re, Top-pair production and decay at NLO matched with parton showers, JHEP 04 (2015) 114 [arXiv:1412.1828] [INSPIRE].

[21] S. Frixione, P. Nason and G. Ridolfi, A positive-weight next-to-leading-order Monte Carlo for heavy flavour hadroproduction, JHEP 09 (2007) 126 [arXiv:0707.3088] [INSPIRE].

[22] T. Sjöstrand et al., An introduction to PYTHIA 8.2, Comput. Phys. Commun. 191 (2015) 159 [arXiv: 1410.3012] [INSPIRE].

[23] P. Skands, S. Carrazza and J. Rojo, Tuning PYTHIA 8.1: the Monash 2013 tune, Eur. Phys. J. C 74 (2014) 3024 [arXiv: 1404.5630] [INSPIRE].

[24] J. Alwall et al., The automated computation of tree-level and next-to-leading order differential cross sections and their matching to parton shower simulations, JHEP 07 (2014) 079 [arXiv: 1405.0301] [INSPIRE].

[25] J. Alwall et al., Comparative study of various algorithms for the merging of parton showers and matrix elements in hadronic collisions, Eur. Phys. J. C 53 (2008) 473 [arXiv:0706.2569] [INSPIRE].

[26] R. Frederix and S. Frixione, Merging meets matching in MC@NLO, JHEP 12 (2012) 061 [arXiv: 1209.6215] [INSPIRE].

[27] E. Re, Single-top Wt-channel production matched with parton showers using the POWHEG method, Eur. Phys. J. C 71 (2011) 1547 [arXiv:1009.2450] [INSPIRE].

[28] S. Alioli, P. Nason, C. Oleari and E. Re, NLO single-top production matched with shower in POWHEG: s- and t-channel contributions, JHEP 09 (2009) 111 [Erratum ibid. 02 (2010) 011] [arXiv:0907.4076] [INSPIRE].

[29] NNPDF collaboration, Parton distributions for the LHC run II, JHEP 04 (2015) 040 [arXiv: 1410.8849] [INSPIRE].

[30] GEANT4 collaboration, GEANT4: a simulation toolkit, Nucl. Instrum. Meth. A 506 (2003) 250 [INSPIRE].

[31] CMS collaboration, Measurement of the inelastic proton-proton cross section at $\sqrt{s}=13 \mathrm{TeV}$, JHEP 07 (2018) 161 [arXiv: 1802. 02613] [INSPIRE]. 
[32] M. Czakon and A. Mitov, Top++: a program for the calculation of the top-pair cross-section at hadron colliders, Comput. Phys. Commun. 185 (2014) 2930 [arXiv:1112.5675] [INSPIRE].

[33] S. Dulat et al., New parton distribution functions from a global analysis of quantum chromodynamics, Phys. Rev. D 93 (2016) 033006 [arXiv: 1506.07443] [INSPIRE].

[34] Y. Li and F. Petriello, Combining QCD and electroweak corrections to dilepton production in FEWZ, Phys. Rev. D 86 (2012) 094034 [arXiv:1208.5967] [INSPIRE].

[35] M. Aliev, H. Lacker, U. Langenfeld, S. Moch, P. Uwer and M. Wiedermann, HATHOR: HAdronic Top and Heavy quarks crOss section calculatoR, Comput. Phys. Commun. 182 (2011) 1034 [arXiv: 1007.1327] [inSPIRE].

[36] P. Kant et al., HatHor for single top-quark production: updated predictions and uncertainty estimates for single top-quark production in hadronic collisions, Comput. Phys. Commun. 191 (2015) 74 [arXiv:1406.4403] [INSPIRE].

[37] N. Kidonakis, Two-loop soft anomalous dimensions for single top quark associated production with $a W^{-}$or $H^{-}$, Phys. Rev. D 82 (2010) 054018 [arXiv: 1005.4451] [inSPIRE].

[38] N. Kidonakis, NNLL threshold resummation for top-pair and single-top production, Phys. Part. Nucl. 45 (2014) 714 [arXiv:1210.7813] [INSPIRE].

[39] J.M. Campbell and R.K. Ellis, MCFM for the Tevatron and the LHC, Nucl. Phys. Proc. Suppl. 205-206 (2010) 10 [arXiv: 1007.3492] [INSPIRE].

[40] J.M. Campbell, R.K. Ellis and C. Williams, Vector boson pair production at the LHC, JHEP 07 (2011) 018 [arXiv: 1105.0020] [inSPIRE].

[41] CMS collaboration, Particle-flow reconstruction and global event description with the CMS detector, 2017 JINST 12 P10003 [arXiv:1706.04965] [INSPIRE].

[42] M. Cacciari, G.P. Salam and G. Soyez, The anti- $k_{t}$ jet clustering algorithm, JHEP 04 (2008) 063 [arXiv: 0802.1189] [INSPIRE].

[43] CMS collaboration, Performance of electron reconstruction and selection with the CMS detector in proton-proton collisions at $\sqrt{s}=8 \mathrm{TeV}, 2015$ JINST $10 \mathrm{P} 06005$ [arXiv: 1502.02701] [INSPIRE].

[44] CMS collaboration, Electron and photon performance in CMS with the full 2016 data sample, CMS-DP-2017-004, CERN, Geneva, Switzerland (2017).

[45] CMS collaboration, Performance of the CMS muon detector and muon reconstruction with proton-proton collisions at $\sqrt{s}=13 \mathrm{TeV}, 2018$ JINST $13 \mathrm{P} 06015$ [arXiv: 1804.04528] [INSPIRE].

[46] CMS collaboration, Performance of reconstruction and identification of $\tau$ leptons decaying to hadrons and $\nu_{\tau}$ in pp collisions at $\sqrt{s}=13 \mathrm{TeV}, 2018$ JINST 13 P10005 [arXiv:1809.02816] [INSPIRE].

[47] CMS collaboration, Identification of heavy-flavour jets with the CMS detector in pp collisions at $13 \mathrm{TeV}, 2018$ JINST $13 \mathrm{P} 05011$ [arXiv:1712.07158] [INSPIRE].

[48] Particle Data Group collaboration, Review of particle physics, Phys. Rev. D 98 (2018) 030001 [INSPIRE].

[49] ATLAS collaboration, Measurement of the inelastic proton-proton cross section at $\sqrt{s}=13$ TeV with the ATLAS detector at the LHC, Phys. Rev. Lett. 117 (2016) 182002 [arXiv: 1606.02625] [INSPIRE].

[50] CMS collaboration, Jet energy scale and resolution in the CMS experiment in pp collisions at $8 \mathrm{TeV}, 2017$ JINST 12 P02014 [arXiv:1607.03663] [INSPIRE]. 
[51] CMS collaboration, CMS luminosity measurements for the 2016 data taking period, CMS-PAS-LUM-17-001, CERN, Geneva, Switzerland (2017).

[52] OPAL collaboration, Inclusive analysis of the $b$ quark fragmentation function in $Z$ decays at LEP, Eur. Phys. J. C 29 (2003) 463 [hep-ex/0210031] [InSPIRE].

[53] ALEPH collaboration, Study of the fragmentation of b quarks into $B$ mesons at the $Z$ peak, Phys. Lett. B 512 (2001) 30 [hep-ex/0106051] [INSPIRE].

[54] SLD collaboration, Measurement of the $b$ quark fragmentation function in $Z^{0}$ decays, Phys. Rev. D 65 (2002) 092006 [Erratum ibid. D 66 (2002) 079905] [hep-ex/0202031] [INSPIRE].

[55] DELPHI collaboration, A study of the b-quark fragmentation function with the DELPHI detector at LEP I and an averaged distribution obtained at the $Z$ pole, Eur. Phys. J. C 71 (2011) 1557 [arXiv: 1102.4748] [INSPIRE].

[56] M.G. Bowler, $e^{+} e^{-}$production of heavy quarks in the string model, Z. Phys. C 11 (1981) 169 [INSPIRE].

[57] C. Peterson, D. Schlatter, I. Schmitt and P.M. Zerwas, Scaling violations in inclusive $e^{+} e^{-}$ annihilation spectra, Phys. Rev. D 27 (1983) 105 [INSPIRE].

[58] CMS collaboration, Measurement of normalized differential t $\bar{t}$ cross sections in the dilepton channel from pp collisions at $\sqrt{s}=13$ TeV, JHEP 04 (2018) 060 [arXiv:1708.07638] [INSPIRE].

[59] CMS collaboration, Measurement of differential cross sections for top quark pair production using the lepton+jets final state in proton-proton collisions at $13 \mathrm{TeV}$, Phys. Rev. D 95 (2017) 092001 [arXiv:1610.04191] [INSPIRE].

[60] CMS collaboration, Measurement of the differential cross section for top quark pair production in pp collisions at $\sqrt{s}=8$ TeV, Eur. Phys. J. C 75 (2015) 542 [arXiv: 1505.04480] [INSPIRE].

[61] CMS collaboration, Measurement of the $t \bar{t}$ production cross section in the all-jets final state in pp collisions at $\sqrt{s}=8$ TeV, Eur. Phys. J. C 76 (2016) 128 [arXiv:1509.06076] [INSPIRE].

[62] CMS collaboration, Investigations of the impact of the parton shower tuning in PYTHIA 8 in the modelling of $t \bar{t}$ at $\sqrt{s}=8$ and 13 TeV, CMS-PAS-TOP-16-021, CERN, Geneva, Switzerland (2016).

[63] ATLAS collaboration, Measurements of the top quark branching ratios into channels with leptons and quarks with the ATLAS detector, Phys. Rev. D 92 (2015) 072005 [arXiv: 1506.05074] [INSPIRE].

[64] CDF collaboration, Study of top-quark production and decays involving a tau lepton at CDF and limits on a charged-Higgs boson contribution, Phys. Rev. D 89 (2014) 091101 [arXiv:1402.6728] [INSPIRE].

[65] CMS collaboration, Measurement of the top quark pair production cross section in pp collisions at $\sqrt{s}=7 \mathrm{TeV}$ in dilepton final states containing a $\tau$, Phys. Rev. D 85 (2012) 112007 [arXiv: 1203.6810] [InSPIRE].

[66] CMS collaboration, Measurement of the $t \bar{t}$ production cross section in pp collisions at $\sqrt{s}=8 \mathrm{TeV}$ in dilepton final states containing one $\tau$ lepton, Phys. Lett. B 739 (2014) 23 [arXiv:1407.6643] [INSPIRE]. 


\section{The CMS collaboration}

\section{Yerevan Physics Institute, Yerevan, Armenia}

A.M. Sirunyan ${ }^{\dagger}$, A. Tumasyan

\section{Institut für Hochenergiephysik, Wien, Austria}

W. Adam, F. Ambrogi, T. Bergauer, J. Brandstetter, M. Dragicevic, J. Erö, A. Escalante Del Valle, M. Flechl, R. Frühwirth ${ }^{1}$, M. Jeitler ${ }^{1}$, N. Krammer, I. Krätschmer, D. Liko, T. Madlener, I. Mikulec, N. Rad, J. Schieck ${ }^{1}$, R. Schöfbeck, M. Spanring, D. Spitzbart, W. Waltenberger, C.-E. Wulz ${ }^{1}$, M. Zarucki

Institute for Nuclear Problems, Minsk, Belarus

V. Drugakov, V. Mossolov, J. Suarez Gonzalez

Universiteit Antwerpen, Antwerpen, Belgium

M.R. Darwish, E.A. De Wolf, D. Di Croce, X. Janssen, A. Lelek, M. Pieters, H. Rejeb Sfar, H. Van Haevermaet, P. Van Mechelen, S. Van Putte, N. Van Remortel

\section{Vrije Universiteit Brussel, Brussel, Belgium}

F. Blekman, E.S. Bols, S.S. Chhibra, J. D'Hondt, J. De Clercq, D. Lontkovskyi, S. Lowette, I. Marchesini, S. Moortgat, Q. Python, K. Skovpen, S. Tavernier, W. Van Doninck, P. Van Mulders

\section{Université Libre de Bruxelles, Bruxelles, Belgium}

D. Beghin, B. Bilin, H. Brun, B. Clerbaux, G. De Lentdecker, H. Delannoy, B. Dorney, L. Favart, A. Grebenyuk, A.K. Kalsi, A. Popov, N. Postiau, E. Starling, L. Thomas, C. Vander Velde, P. Vanlaer, D. Vannerom

\section{Ghent University, Ghent, Belgium}

T. Cornelis, D. Dobur, I. Khvastunov ${ }^{2}$, M. Niedziela, C. Roskas, M. Tytgat, W. Verbeke, B. Vermassen, M. Vit

\section{Université Catholique de Louvain, Louvain-la-Neuve, Belgium}

O. Bondu, G. Bruno, C. Caputo, P. David, C. Delaere, M. Delcourt, A. Giammanco, V. Lemaitre, J. Prisciandaro, A. Saggio, M. Vidal Marono, P. Vischia, J. Zobec

Centro Brasileiro de Pesquisas Fisicas, Rio de Janeiro, Brazil

F.L. Alves, G.A. Alves, G. Correia Silva, C. Hensel, A. Moraes, P. Rebello Teles

Universidade do Estado do Rio de Janeiro, Rio de Janeiro, Brazil

E. Belchior Batista Das Chagas, W. Carvalho, J. Chinellato ${ }^{3}$, E. Coelho, E.M. Da Costa, G.G. Da Silveira ${ }^{4}$, D. De Jesus Damiao, C. De Oliveira Martins, S. Fonseca De Souza, L.M. Huertas Guativa, H. Malbouisson, J. Martins ${ }^{5}$, D. Matos Figueiredo, M. Medina Jaime ${ }^{6}$, M. Melo De Almeida, C. Mora Herrera, L. Mundim, H. Nogima, W.L. Prado Da Silva, L.J. Sanchez Rosas, A. Santoro, A. Sznajder, M. Thiel, E.J. Tonelli Manganote ${ }^{3}$, F. Torres Da Silva De Araujo, A. Vilela Pereira 
Universidade Estadual Paulista ${ }^{a}$, Universidade Federal do $\mathrm{ABC}^{b}$, São Paulo, Brazil

C.A. Bernardes ${ }^{a}$, L. Calligaris ${ }^{a}$, T.R. Fernandez Perez Tomei ${ }^{a}$, E.M. Gregores ${ }^{b}$, D.S. Lemos, P.G. Mercadante ${ }^{b}$, S.F. Novaes ${ }^{a}$, SandraS. Padula ${ }^{a}$

Institute for Nuclear Research and Nuclear Energy, Bulgarian Academy of Sciences, Sofia, Bulgaria

A. Aleksandrov, G. Antchev, R. Hadjiiska, P. Iaydjiev, M. Misheva, M. Rodozov, M. Shopova, G. Sultanov

University of Sofia, Sofia, Bulgaria

M. Bonchev, A. Dimitrov, T. Ivanov, L. Litov, B. Pavlov, P. Petkov

Beihang University, Beijing, China

W. Fang ${ }^{7}$, X. Gao ${ }^{7}$, L. Yuan

Institute of High Energy Physics, Beijing, China

G.M. Chen, H.S. Chen, M. Chen, C.H. Jiang, D. Leggat, H. Liao, Z. Liu, A. Spiezia, J. Tao,

E. Yazgan, H. Zhang, S. Zhang ${ }^{8}$, J. Zhao

State Key Laboratory of Nuclear Physics and Technology, Peking University, Beijing, China

A. Agapitos, Y. Ban, G. Chen, A. Levin, J. Li, L. Li, Q. Li, Y. Mao, S.J. Qian, D. Wang, Q. Wang

Tsinghua University, Beijing, China

M. Ahmad, Z. Hu, Y. Wang

Zhejiang University, Hangzhou, China

M. Xiao

Universidad de Los Andes, Bogota, Colombia

C. Avila, A. Cabrera, C. Florez, C.F. González Hernández, M.A. Segura Delgado

Universidad de Antioquia, Medellin, Colombia

J. Mejia Guisao, J.D. Ruiz Alvarez, C.A. Salazar González, N. Vanegas Arbelaez

University of Split, Faculty of Electrical Engineering, Mechanical Engineering and Naval Architecture, Split, Croatia

D. Giljanović, N. Godinovic, D. Lelas, I. Puljak, T. Sculac

University of Split, Faculty of Science, Split, Croatia

Z. Antunovic, M. Kovac

Institute Rudjer Boskovic, Zagreb, Croatia

V. Brigljevic, D. Ferencek, K. Kadija, B. Mesic, M. Roguljic, A. Starodumov ${ }^{9}$, T. Susa

University of Cyprus, Nicosia, Cyprus

M.W. Ather, A. Attikis, E. Erodotou, A. Ioannou, M. Kolosova, S. Konstantinou,

G. Mavromanolakis, J. Mousa, C. Nicolaou, F. Ptochos, P.A. Razis, H. Rykaczewski,

D. Tsiakkouri 
Charles University, Prague, Czech Republic

M. Finger ${ }^{10}$, M. Finger Jr. ${ }^{10}$, A. Kveton, J. Tomsa

Escuela Politecnica Nacional, Quito, Ecuador

E. Ayala

Universidad San Francisco de Quito, Quito, Ecuador

E. Carrera Jarrin

Academy of Scientific Research and Technology of the Arab Republic of Egypt, Egyptian Network of High Energy Physics, Cairo, Egypt

A.A. Abdelalim ${ }^{11,12}$, S. Abu Zeid

National Institute of Chemical Physics and Biophysics, Tallinn, Estonia

S. Bhowmik, A. Carvalho Antunes De Oliveira, R.K. Dewanjee, K. Ehataht, M. Kadastik, M. Raidal, C. Veelken

Department of Physics, University of Helsinki, Helsinki, Finland

P. Eerola, L. Forthomme, H. Kirschenmann, K. Osterberg, M. Voutilainen

Helsinki Institute of Physics, Helsinki, Finland

F. Garcia, J. Havukainen, J.K. Heikkilä, V. Karimäki, M.S. Kim, R. Kinnunen, T. Lampén,

K. Lassila-Perini, S. Laurila, S. Lehti, T. Lindén, P. Luukka, T. Mäenpää, H. Siikonen,

E. Tuominen, J. Tuominiemi

Lappeenranta University of Technology, Lappeenranta, Finland

T. Tuuva

IRFU, CEA, Université Paris-Saclay, Gif-sur-Yvette, France

M. Besancon, F. Couderc, M. Dejardin, D. Denegri, B. Fabbro, J.L. Faure, F. Ferri,

S. Ganjour, A. Givernaud, P. Gras, G. Hamel de Monchenault, P. Jarry, C. Leloup,

B. Lenzi, E. Locci, J. Malcles, J. Rander, A. Rosowsky, M.Ö. Sahin, A. Savoy-Navarro ${ }^{13}$,

M. Titov, G.B. Yu

Laboratoire Leprince-Ringuet, CNRS/IN2P3, Ecole Polytechnique, Institut Polytechnique de Paris

S. Ahuja, C. Amendola, F. Beaudette, P. Busson, C. Charlot, B. Diab, G. Falmagne,

R. Granier de Cassagnac, I. Kucher, A. Lobanov, C. Martin Perez, M. Nguyen, C. Ochando,

P. Paganini, J. Rembser, R. Salerno, J.B. Sauvan, Y. Sirois, A. Zabi, A. Zghiche

Université de Strasbourg, CNRS, IPHC UMR 7178, Strasbourg, France

J.-L. Agram ${ }^{14}$, J. Andrea, D. Bloch, G. Bourgatte, J.-M. Brom, E.C. Chabert, C. Collard,

E. Conte ${ }^{14}$, J.-C. Fontaine ${ }^{14}$, D. Gelé, U. Goerlach, M. Jansová, A.-C. Le Bihan, N. Tonon,

P. Van Hove

Centre de Calcul de l'Institut National de Physique Nucleaire et de Physique des Particules, CNRS/IN2P3, Villeurbanne, France

S. Gadrat 
Université de Lyon, Université Claude Bernard Lyon 1, CNRS-IN2P3, Institut de Physique Nucléaire de Lyon, Villeurbanne, France

S. Beauceron, C. Bernet, G. Boudoul, C. Camen, A. Carle, N. Chanon, R. Chierici, D. Contardo, P. Depasse, H. El Mamouni, J. Fay, S. Gascon, M. Gouzevitch, B. Ille, Sa. Jain, F. Lagarde, I.B. Laktineh, H. Lattaud, A. Lesauvage, M. Lethuillier, L. Mirabito, S. Perries, V. Sordini, L. Torterotot, G. Touquet, M. Vander Donckt, S. Viret

Georgian Technical University, Tbilisi, Georgia

T. Toriashvili ${ }^{15}$

Tbilisi State University, Tbilisi, Georgia

Z. Tsamalaidze ${ }^{10}$

RWTH Aachen University, I. Physikalisches Institut, Aachen, Germany

C. Autermann, L. Feld, M.K. Kiesel, K. Klein, M. Lipinski, D. Meuser, A. Pauls, M. Preuten, M.P. Rauch, J. Schulz, M. Teroerde, B. Wittmer

RWTH Aachen University, III. Physikalisches Institut A, Aachen, Germany

M. Erdmann, B. Fischer, S. Ghosh, T. Hebbeker, K. Hoepfner, H. Keller, L. Mastrolorenzo, M. Merschmeyer, A. Meyer, P. Millet, G. Mocellin, S. Mondal, S. Mukherjee, D. Noll, A. Novak, T. Pook, A. Pozdnyakov, T. Quast, M. Radziej, Y. Rath, H. Reithler, J. Roemer, A. Schmidt, S.C. Schuler, A. Sharma, S. Wiedenbeck, S. Zaleski

RWTH Aachen University, III. Physikalisches Institut B, Aachen, Germany

G. Flügge, W. Haj Ahmad ${ }^{16}$, O. Hlushchenko, T. Kress, T. Müller, A. Nowack, C. Pistone, O. Pooth, D. Roy, H. Sert, A. Stahl ${ }^{17}$

\section{Deutsches Elektronen-Synchrotron, Hamburg, Germany}

M. Aldaya Martin, P. Asmuss, I. Babounikau, H. Bakhshiansohi, K. Beernaert, O. Behnke, A. Bermúdez Martínez, D. Bertsche, A.A. Bin Anuar, K. Borras ${ }^{18}$, V. Botta, A. Campbell, A. Cardini, P. Connor, S. Consuegra Rodríguez, C. Contreras-Campana, V. Danilov, A. De Wit, M.M. Defranchis, C. Diez Pardos, D. Domínguez Damiani, G. Eckerlin, D. Eckstein, T. Eichhorn, A. Elwood, E. Eren, E. Gallo ${ }^{19}$, A. Geiser, A. Grohsjean, M. Guthoff, M. Haranko, A. Harb, A. Jafari, N.Z. Jomhari, H. Jung, A. Kasem ${ }^{18}$, M. Kasemann, H. Kaveh, J. Keaveney, C. Kleinwort, J. Knolle, D. Krücker, W. Lange, T. Lenz, J. Lidrych, K. Lipka, W. Lohmann ${ }^{20}$, R. Mankel, I.-A. Melzer-Pellmann, A.B. Meyer, M. Meyer, M. Missiroli, G. Mittag, J. Mnich, A. Mussgiller, V. Myronenko, D. Pérez Adán, S.K. Pflitsch, D. Pitzl, A. Raspereza, A. Saibel, M. Savitskyi, V. Scheurer, P. Schütze, C. Schwanenberger, R. Shevchenko, A. Singh, H. Tholen, O. Turkot, A. Vagnerini, M. Van De Klundert, R. Walsh, Y. Wen, K. Wichmann, C. Wissing, O. Zenaiev, R. Zlebcik

\section{University of Hamburg, Hamburg, Germany}

R. Aggleton, S. Bein, L. Benato, A. Benecke, V. Blobel, T. Dreyer, A. Ebrahimi, F. Feindt, A. Fröhlich, C. Garbers, E. Garutti, D. Gonzalez, P. Gunnellini, J. Haller, A. Hinzmann, A. Karavdina, G. Kasieczka, R. Klanner, R. Kogler, N. Kovalchuk, S. Kurz, V. Kutzner, J. Lange, T. Lange, A. Malara, J. Multhaup, C.E.N. Niemeyer, A. Perieanu, A. Reimers, 
O. Rieger, C. Scharf, P. Schleper, S. Schumann, J. Schwandt, J. Sonneveld, H. Stadie, G. Steinbrück, F.M. Stober, B. Vormwald, I. Zoi

Karlsruher Institut fuer Technologie, Karlsruhe, Germany

M. Akbiyik, C. Barth, M. Baselga, S. Baur, T. Berger, E. Butz, R. Caspart, T. Chwalek, W. De Boer, A. Dierlamm, K. El Morabit, N. Faltermann, M. Giffels, P. Goldenzweig, A. Gottmann, M.A. Harrendorf, F. Hartmann ${ }^{17}$, U. Husemann, S. Kudella, S. Mitra, M.U. Mozer, D. Müller, Th. Müller, M. Musich, A. Nürnberg, G. Quast, K. Rabbertz, M. Schröder, I. Shvetsov, H.J. Simonis, R. Ulrich, M. Wassmer, M. Weber, C. Wöhrmann, R. Wolf

Institute of Nuclear and Particle Physics (INPP), NCSR Demokritos, Aghia Paraskevi, Greece

G. Anagnostou, P. Asenov, G. Daskalakis, T. Geralis, A. Kyriakis, D. Loukas, G. Paspalaki National and Kapodistrian University of Athens, Athens, Greece

M. Diamantopoulou, G. Karathanasis, P. Kontaxakis, A. Manousakis-katsikakis, A. Panagiotou, I. Papavergou, N. Saoulidou, A. Stakia, K. Theofilatos, K. Vellidis, E. Vourliotis

National Technical University of Athens, Athens, Greece

G. Bakas, K. Kousouris, I. Papakrivopoulos, G. Tsipolitis

University of Ioánnina, Ioánnina, Greece

I. Evangelou, C. Foudas, P. Gianneios, P. Katsoulis, P. Kokkas, S. Mallios, K. Manitara, N. Manthos, I. Papadopoulos, J. Strologas, F.A. Triantis, D. Tsitsonis

MTA-ELTE Lendület CMS Particle and Nuclear Physics Group, Eötvös Loránd University, Budapest, Hungary

M. Bartók ${ }^{21}$, R. Chudasama, M. Csanad, P. Major, K. Mandal, A. Mehta, M.I. Nagy, G. Pasztor, O. Surányi, G.I. Veres

Wigner Research Centre for Physics, Budapest, Hungary

G. Bencze, C. Hajdu, D. Horvath ${ }^{22}$, F. Sikler, T.Á. Vámi, V. Veszpremi, G. Vesztergombi ${ }^{\dagger}$

Institute of Nuclear Research ATOMKI, Debrecen, Hungary

N. Beni, S. Czellar, J. Karancsi ${ }^{21}$, A. Makovec, J. Molnar, Z. Szillasi

Institute of Physics, University of Debrecen, Debrecen, Hungary

P. Raics, D. Teyssier, Z.L. Trocsanyi, B. Ujvari

Eszterhazy Karoly University, Karoly Robert Campus, Gyongyos, Hungary

T. Csorgo, W.J. Metzger, F. Nemes, T. Novak

Indian Institute of Science (IISc), Bangalore, India

S. Choudhury, J.R. Komaragiri, P.C. Tiwari

National Institute of Science Education and Research, HBNI, Bhubaneswar, India

S. Bahinipati ${ }^{24}$, C. Kar, G. Kole, P. Mal, V.K. Muraleedharan Nair Bindhu, A. Nayak ${ }^{25}$, D.K. Sahoo ${ }^{24}$, S.K. Swain 
Panjab University, Chandigarh, India

S. Bansal, S.B. Beri, V. Bhatnagar, S. Chauhan, R. Chawla, N. Dhingra, R. Gupta, A. Kaur, M. Kaur, S. Kaur, P. Kumari, M. Lohan, M. Meena, K. Sandeep, S. Sharma, J.B. Singh, A.K. Virdi, G. Walia

University of Delhi, Delhi, India

A. Bhardwaj, B.C. Choudhary, R.B. Garg, M. Gola, S. Keshri, Ashok Kumar, M. Naimuddin, P. Priyanka, K. Ranjan, Aashaq Shah, R. Sharma

Saha Institute of Nuclear Physics, HBNI, Kolkata, India

R. Bhardwaj ${ }^{26}$, M. Bharti ${ }^{26}$, R. Bhattacharya, S. Bhattacharya, U. Bhawandeep ${ }^{26}$,

D. Bhowmik, S. Dutta, S. Ghosh, B. Gomber ${ }^{27}$, M. Maity ${ }^{28}$, K. Mondal, S. Nandan, A. Purohit, P.K. Rout, G. Saha, S. Sarkar, T. Sarkar ${ }^{28}$, M. Sharan, B. Singh ${ }^{26}$, S. Thakur ${ }^{26}$

Indian Institute of Technology Madras, Madras, India

P.K. Behera, P. Kalbhor, A. Muhammad, P.R. Pujahari, A. Sharma, A.K. Sikdar

Bhabha Atomic Research Centre, Mumbai, India

D. Dutta, V. Jha, V. Kumar, D.K. Mishra, P.K. Netrakanti, L.M. Pant, P. Shukla

Tata Institute of Fundamental Research-A, Mumbai, India

T. Aziz, M.A. Bhat, S. Dugad, G.B. Mohanty, N. Sur, RavindraKumar Verma

Tata Institute of Fundamental Research-B, Mumbai, India

S. Banerjee, S. Bhattacharya, S. Chatterjee, P. Das, M. Guchait, S. Karmakar, S. Kumar, G. Majumder, K. Mazumdar, N. Sahoo, S. Sawant

Indian Institute of Science Education and Research (IISER), Pune, India

S. Dube, B. Kansal, A. Kapoor, K. Kothekar, S. Pandey, A. Rane, A. Rastogi, S. Sharma

Institute for Research in Fundamental Sciences (IPM), Tehran, Iran

S. Chenarani ${ }^{29}$, E. Eskandari Tadavani, S.M. Etesami ${ }^{29}$, M. Khakzad, M. Mohammadi Najafabadi, M. Naseri, F. Rezaei Hosseinabadi

University College Dublin, Dublin, Ireland

M. Felcini, M. Grunewald

INFN Sezione di Bari ${ }^{a}$, Università di Bari ${ }^{b}$, Politecnico di Bari ${ }^{c}$, Bari, Italy M. Abbrescia ${ }^{a, b}$, R. Aly ${ }^{a, b, 30}$, C. Calabria ${ }^{a, b}$, A. Colaleo $^{a}$, D. Creanza $^{a, c}$, L. Cristella $^{a, b}$, N. De Filippis ${ }^{a, c}$, M. De Palma ${ }^{a, b}$, A. Di Florio ${ }^{a, b}$, W. Elmetenawee ${ }^{a, b}$, L. Fiore ${ }^{a}$,

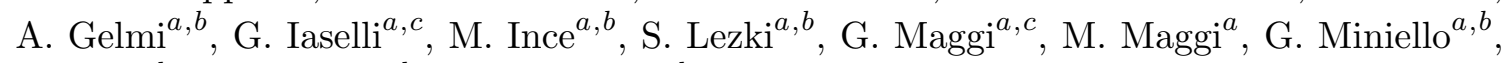
S. $\mathrm{My}^{a, b}$, S. Nuzzo ${ }^{a, b}$, A. Pompili ${ }^{a, b}$, G. Pugliese ${ }^{a, c}$, R. Radogna ${ }^{a}$, A. Ranieri ${ }^{a}$,

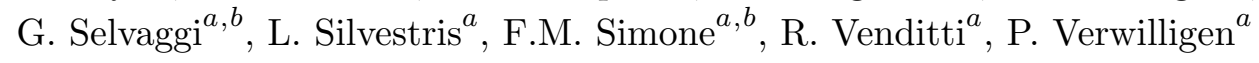

INFN Sezione di Bologna ${ }^{a}$, Università di Bologna ${ }^{b}$, Bologna, Italy

G. Abbiendi ${ }^{a}$, C. Battilana ${ }^{a, b}$, D. Bonacorsi ${ }^{a, b}$, L. Borgonovi $^{a, b}$, S. Braibant-Giacomelli ${ }^{a, b}$,

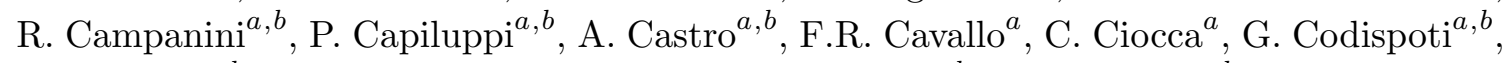

M. Cuffiani ${ }^{a, b}$, G.M. Dallavalle ${ }^{a}$, F. Fabbri ${ }^{a}$, A. Fanfani ${ }^{a, b}$, E. Fontanesi $^{a, b}$, P. Giacomelli $^{a}$,

C. Grandi ${ }^{a}$, L. Guiducci ${ }^{a, b}$, F. Iemmi ${ }^{a, b}$, S. Lo Meo ${ }^{a, 31}$, S. Marcellini ${ }^{a}$, G. Masetti ${ }^{a}$, 


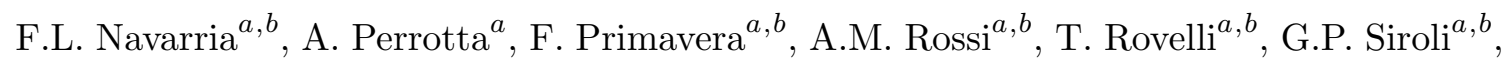
N. $\operatorname{Tosi}^{a}$

INFN Sezione di Catania ${ }^{a}$, Università di Catania ${ }^{b}$, Catania, Italy

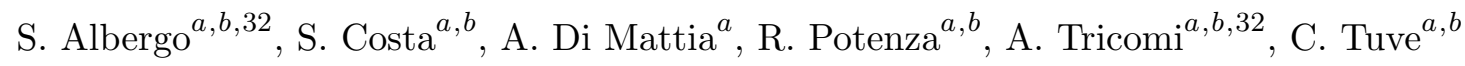

INFN Sezione di Firenze ${ }^{a}$, Università di Firenze ${ }^{b}$, Firenze, Italy

G. Barbagli ${ }^{a}$, A. Cassese, R. Ceccarelli, V. Ciulli ${ }^{a, b}$, C. Civinini $^{a}$, R. D’Alessandro ${ }^{a, b}$,

F. Fiori ${ }^{a, c}$, E. Focardi ${ }^{a, b}$, G. Latino ${ }^{a, b}$, P. Lenzi ${ }^{a, b}$, M. Meschini ${ }^{a}$, S. Paoletti ${ }^{a}$,

G. Sguazzoni ${ }^{a}$, L. Viliani ${ }^{a}$

INFN Laboratori Nazionali di Frascati, Frascati, Italy

L. Benussi, S. Bianco, D. Piccolo

INFN Sezione di Genova ${ }^{a}$, Università di Genova ${ }^{b}$, Genova, Italy

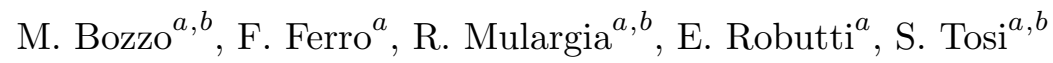

INFN Sezione di Milano-Bicocca ${ }^{a}$, Università di Milano-Bicocca ${ }^{b}$, Milano, Italy

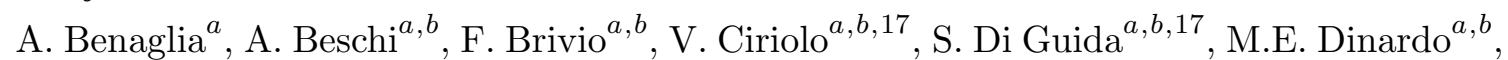

P. Dini ${ }^{a}$, S. Gennai ${ }^{a}$, A. Ghezzi ${ }^{a, b}$, P. Govoni ${ }^{a}, b$, L. Guzzi ${ }^{a} b$, M. Malberti $^{a}$, S. Malvezzi ${ }^{a}$,

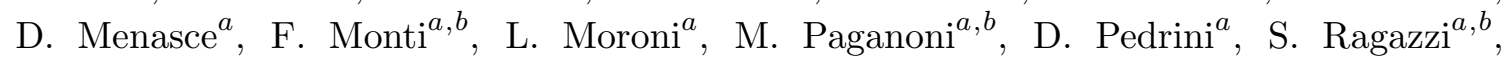

T. Tabarelli de Fatis ${ }^{a, b}$, D. Zuolo ${ }^{a, b}$

INFN Sezione di Napoli ${ }^{a}$, Università di Napoli 'Federico II' ${ }^{b}$, Napoli, Italy, Università della Basilicata $^{c}$, Potenza, Italy, Università G. Marconi ${ }^{d}$, Roma, Italy

S. Buontempo ${ }^{a}$, N. Cavallo ${ }^{a, c}$, A. De Iorio ${ }^{a, b}$, A. Di Crescenzo ${ }^{a, b}$, F. Fabozzi $^{a, c}$, F. Fienga $^{a}$,

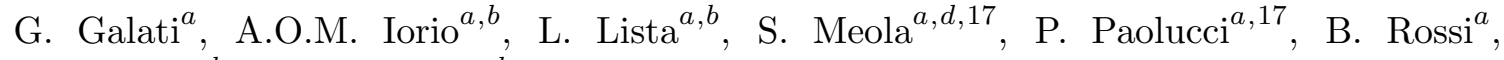
C. Sciacca ${ }^{a, b}$, E. Voevodina ${ }^{a, b}$

INFN Sezione di Padova ${ }^{a}$, Università di Padova ${ }^{b}$, Padova, Italy, Università di Trento ${ }^{c}$, Trento, Italy

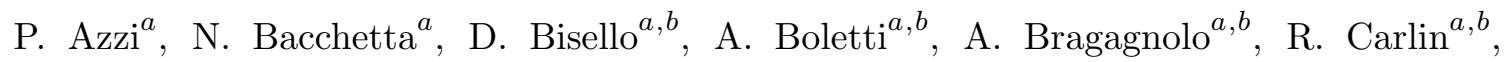

P. Checchia ${ }^{a}$, P. De Castro Manzano ${ }^{a}$, T. Dorigo ${ }^{a}$, U. Dosselli ${ }^{a}$, F. Gasparini ${ }^{a, b}{ }^{\prime}$

U. Gasparini ${ }^{a, b}$, A. Gozzelino ${ }^{a}$, S.Y. Hoh $^{a, b}$, P. Lujan ${ }^{a}$, M. Margoni $^{a, b}$, A.T. Meneguzzo ${ }^{a, b}$,

J. Pazzini ${ }^{a, b}$, M. Presilla ${ }^{b}$, P. Ronchese ${ }^{a, b}$, R. Rossin ${ }^{a, b}$, F. Simonetto ${ }^{a, b}$, A. Tiko ${ }^{a}$, M. Tosi ${ }^{a, b}$, M. Zanetti ${ }^{a, b}$, P. Zotto ${ }^{a, b}$, G. Zumerle ${ }^{a, b}$

INFN Sezione di Pavia ${ }^{a}$, Università di Pavia ${ }^{b}$, Pavia, Italy

A. Braghieri ${ }^{a}$, D. Fiorina ${ }^{a, b}$, P. Montagna ${ }^{a, b}$, S.P. Ratti ${ }^{a, b}$, V. $\operatorname{Re}^{a}$, M. Ressegotti ${ }^{a, b}$,

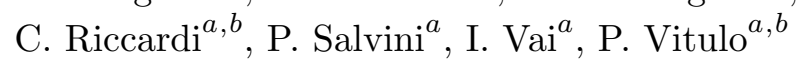

INFN Sezione di Perugia ${ }^{a}$, Università di Perugia ${ }^{b}$, Perugia, Italy

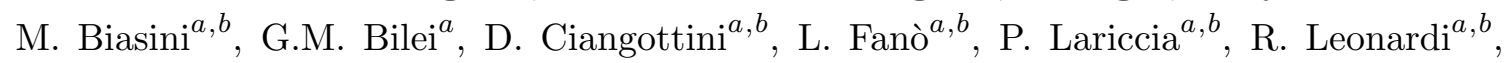

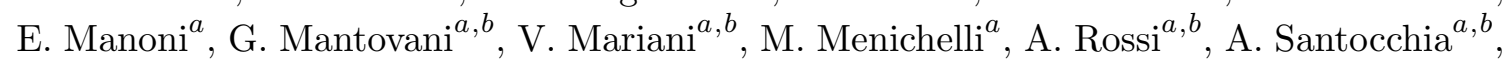

D. Spiga ${ }^{a}$ 
INFN Sezione di Pisa ${ }^{a}$, Università di Pisa ${ }^{b}$, Scuola Normale Superiore di Pisa c, Pisa, Italy

K. Androsov $^{a}$, P. Azzurri ${ }^{a}$, G. Bagliesi ${ }^{a}$, V. Bertacchi ${ }^{a, c}$, L. Bianchini $^{a}$, T. Boccali ${ }^{a}$,

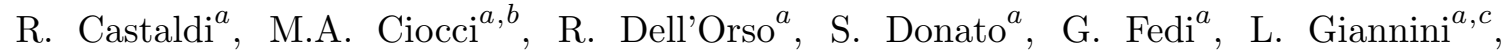

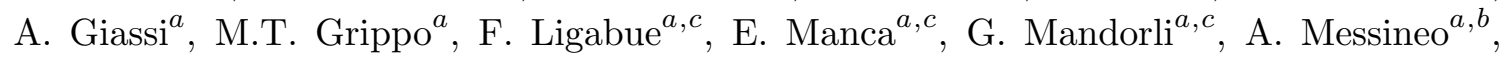
F. Palla ${ }^{a}$, A. Rizzi ${ }^{a}, b$, G. Rolandi ${ }^{33}$, S. Roy Chowdhury, A. Scribano ${ }^{a}$, P. Spagnolo ${ }^{a}$,

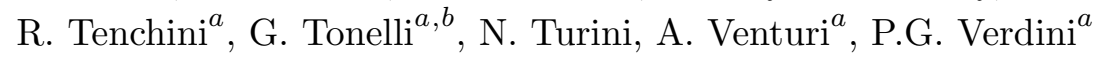

INFN Sezione di Roma ${ }^{a}$, Sapienza Università di Roma ${ }^{b}$, Rome, Italy

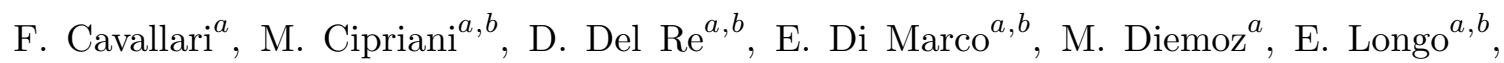
P. Meridiani ${ }^{a}$, G. Organtini ${ }^{a, b}$, F. Pandolfi ${ }^{a}$, R. Paramatti ${ }^{a, b}$, C. Quaranta ${ }^{a, b}$, S. Rahatlou ${ }^{a, b}$, C. Rovelli ${ }^{a}$, F. Santanastasio ${ }^{a, b}$, L. Soffi $^{a, b}$

INFN Sezione di Torino ${ }^{a}$, Università di Torino ${ }^{b}$, Torino, Italy, Università del Piemonte Orientale ${ }^{c}$, Novara, Italy

N. Amapane ${ }^{a, b}$, R. Arcidiacono ${ }^{a, c}, \mathrm{~S}$. Argiro ${ }^{a, b}$, M. Arneodo $^{a, c}$, N. Bartosik ${ }^{a}$, R. Bellan $^{a, b}$, A. Bellora, C. Biino ${ }^{a}$, A. Cappati ${ }^{a, b}$, N. Cartiglia ${ }^{a}$, S. Cometti ${ }^{a}$, M. Costa $^{a, b}$,

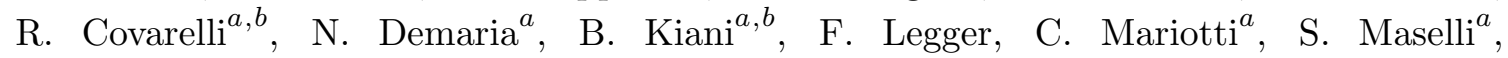
E. Migliore $^{a, b}$, V. Monaco ${ }^{a, b}$, E. Monteil ${ }^{a, b}$, M. Monteno ${ }^{a}$, M.M. Obertino ${ }^{a, b}$, G. Ortona $^{a, b}$, L. Pacher ${ }^{a, b}$, N. Pastrone ${ }^{a}$, M. Pelliccioni ${ }^{a}$, G.L. Pinna Angioni ${ }^{a, b}$, A. Romero ${ }^{a, b}$,

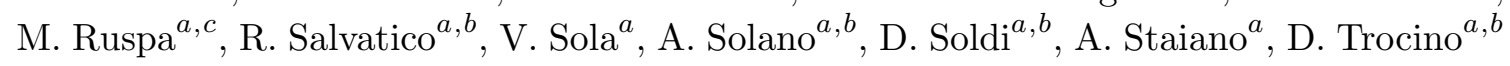
INFN Sezione di Trieste ${ }^{a}$, Università di Trieste ${ }^{b}$, Trieste, Italy

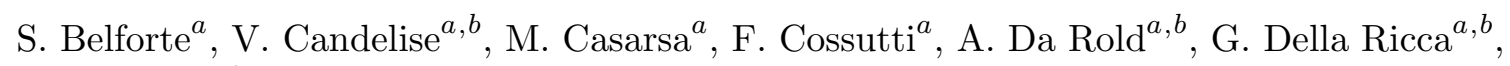
F. Vazzoler ${ }^{a, b}$, A. Zanetti ${ }^{a}$

Kyungpook National University, Daegu, Korea

B. Kim, D.H. Kim, G.N. Kim, J. Lee, S.W. Lee, C.S. Moon, Y.D. Oh, S.I. Pak, S. Sekmen, D.C. Son, Y.C. Yang

Chonnam National University, Institute for Universe and Elementary Particles, Kwangju, Korea

H. Kim, D.H. Moon, G. Oh

Hanyang University, Seoul, Korea

B. Francois, T.J. Kim, J. Park

Korea University, Seoul, Korea

S. Cho, S. Choi, Y. Go, S. Ha, B. Hong, K. Lee, K.S. Lee, J. Lim, J. Park, S.K. Park, Y. Roh, J. Yoo

Kyung Hee University, Department of Physics

J. Goh

Sejong University, Seoul, Korea

H.S. Kim 
Seoul National University, Seoul, Korea

J. Almond, J.H. Bhyun, J. Choi, S. Jeon, J. Kim, J.S. Kim, H. Lee, K. Lee, S. Lee, K. Nam, M. Oh, S.B. Oh, B.C. Radburn-Smith, U.K. Yang, H.D. Yoo, I. Yoon

University of Seoul, Seoul, Korea

D. Jeon, J.H. Kim, J.S.H. Lee, I.C. Park, I.J Watson

Sungkyunkwan University, Suwon, Korea

Y. Choi, C. Hwang, Y. Jeong, J. Lee, Y. Lee, I. Yu

Riga Technical University, Riga, Latvia

V. Veckalns ${ }^{34}$

Vilnius University, Vilnius, Lithuania

V. Dudenas, A. Juodagalvis, A. Rinkevicius, G. Tamulaitis, J. Vaitkus

National Centre for Particle Physics, Universiti Malaya, Kuala Lumpur, Malaysia

Z.A. Ibrahim, F. Mohamad Idris ${ }^{35}$, W.A.T. Wan Abdullah, M.N. Yusli, Z. Zolkapli

Universidad de Sonora (UNISON), Hermosillo, Mexico

J.F. Benitez, A. Castaneda Hernandez, J.A. Murillo Quijada, L. Valencia Palomo

Centro de Investigacion y de Estudios Avanzados del IPN, Mexico City, Mexico

H. Castilla-Valdez, E. De La Cruz-Burelo, I. Heredia-De La Cruz ${ }^{36}$, R. Lopez-Fernandez,

A. Sanchez-Hernandez

Universidad Iberoamericana, Mexico City, Mexico

S. Carrillo Moreno, C. Oropeza Barrera, M. Ramirez-Garcia, F. Vazquez Valencia

Benemerita Universidad Autonoma de Puebla, Puebla, Mexico

J. Eysermans, I. Pedraza, H.A. Salazar Ibarguen, C. Uribe Estrada

Universidad Autónoma de San Luis Potosí, San Luis Potosí, Mexico

A. Morelos Pineda

University of Montenegro, Podgorica, Montenegro

J. Mijuskovic ${ }^{2}$, N. Raicevic

University of Auckland, Auckland, New Zealand

D. Krofcheck

University of Canterbury, Christchurch, New Zealand

S. Bheesette, P.H. Butler

National Centre for Physics, Quaid-I-Azam University, Islamabad, Pakistan

A. Ahmad, M. Ahmad, Q. Hassan, H.R. Hoorani, W.A. Khan, M.A. Shah, M. Shoaib, M. Waqas

AGH University of Science and Technology Faculty of Computer Science, Electronics and Telecommunications, Krakow, Poland

V. Avati, L. Grzanka, M. Malawski 
National Centre for Nuclear Research, Swierk, Poland

H. Bialkowska, M. Bluj, B. Boimska, M. Górski, M. Kazana, M. Szleper, P. Zalewski

Institute of Experimental Physics, Faculty of Physics, University of Warsaw, Warsaw, Poland

K. Bunkowski, A. Byszuk ${ }^{37}$, K. Doroba, A. Kalinowski, M. Konecki, J. Krolikowski, M. Olszewski, M. Walczak

Laboratório de Instrumentação e Física Experimental de Partículas, Lisboa, Portugal

M. Araujo, P. Bargassa, D. Bastos, A. Di Francesco, P. Faccioli, B. Galinhas, M. Gallinaro,

J. Hollar, N. Leonardo, T. Niknejad, J. Seixas, K. Shchelina, G. Strong, O. Toldaiev,

J. Varela

Joint Institute for Nuclear Research, Dubna, Russia

S. Afanasiev, P. Bunin, M. Gavrilenko, I. Golutvin, I. Gorbunov, A. Kamenev, V. Karjavine, A. Lanev, A. Malakhov, V. Matveev ${ }^{38,39}$, P. Moisenz, V. Palichik, V. Perelygin, M. Savina, S. Shmatov, S. Shulha, N. Skatchkov, V. Smirnov, N. Voytishin, A. Zarubin

Petersburg Nuclear Physics Institute, Gatchina (St. Petersburg), Russia

L. Chtchipounov, V. Golovtcov, Y. Ivanov, V. Kim ${ }^{40}$, E. Kuznetsova ${ }^{41}$, P. Levchenko, V. Murzin, V. Oreshkin, I. Smirnov, D. Sosnov, V. Sulimov, L. Uvarov, A. Vorobyev

Institute for Nuclear Research, Moscow, Russia

Yu. Andreev, A. Dermenev, S. Gninenko, N. Golubev, A. Karneyeu, M. Kirsanov, N. Krasnikov, A. Pashenkov, D. Tlisov, A. Toropin

Institute for Theoretical and Experimental Physics named by A.I. Alikhanov of NRC 'Kurchatov Institute', Moscow, Russia

V. Epshteyn, V. Gavrilov, N. Lychkovskaya, A. Nikitenko ${ }^{42}$, V. Popov, I. Pozdnyakov, G. Safronov, A. Spiridonov, A. Stepennov, M. Toms, E. Vlasov, A. Zhokin

Moscow Institute of Physics and Technology, Moscow, Russia

T. Aushev

National Research Nuclear University 'Moscow Engineering Physics Institute' (MEPhI), Moscow, Russia

M. Chadeeva ${ }^{43}$, P. Parygin, D. Philippov, E. Popova, V. Rusinov

P.N. Lebedev Physical Institute, Moscow, Russia

V. Andreev, M. Azarkin, I. Dremin, M. Kirakosyan, A. Terkulov

Skobeltsyn Institute of Nuclear Physics, Lomonosov Moscow State University, Moscow, Russia

A. Baskakov, A. Belyaev, E. Boos, V. Bunichev, M. Dubinin ${ }^{44}$, L. Dudko, V. Klyukhin,

N. Korneeva, I. Lokhtin, S. Obraztsov, M. Perfilov, V. Savrin, P. Volkov

Novosibirsk State University (NSU), Novosibirsk, Russia

A. Barnyakov ${ }^{45}$, V. Blinov ${ }^{45}$, T. Dimova ${ }^{45}$, L. Kardapoltsev ${ }^{45}$, Y. Skovpen ${ }^{45}$ 
Institute for High Energy Physics of National Research Centre 'Kurchatov Institute', Protvino, Russia

I. Azhgirey, I. Bayshev, S. Bitioukov, V. Kachanov, D. Konstantinov, P. Mandrik, V. Petrov, R. Ryutin, S. Slabospitskii, A. Sobol, S. Troshin, N. Tyurin, A. Uzunian, A. Volkov

National Research Tomsk Polytechnic University, Tomsk, Russia

A. Babaev, A. Iuzhakov, V. Okhotnikov

Tomsk State University, Tomsk, Russia

V. Borchsh, V. Ivanchenko, E. Tcherniaev

University of Belgrade: Faculty of Physics and VINCA Institute of Nuclear Sciences

P. Adzic ${ }^{46}$, P. Cirkovic, M. Dordevic, P. Milenovic, J. Milosevic, M. Stojanovic

Centro de Investigaciones Energéticas Medioambientales y Tecnológicas (CIEMAT), Madrid, Spain

M. Aguilar-Benitez, J. Alcaraz Maestre, A. Álvarez Fernández, I. Bachiller, M. Barrio Luna, CristinaF. Bedoya, J.A. Brochero Cifuentes, C.A. Carrillo Montoya, M. Cepeda, M. Cerrada, N. Colino, B. De La Cruz, A. Delgado Peris, J.P. Fernández Ramos, J. Flix, M.C. Fouz, O. Gonzalez Lopez, S. Goy Lopez, J.M. Hernandez, M.I. Josa, D. Moran, Á. Navarro Tobar, A. Pérez-Calero Yzquierdo, J. Puerta Pelayo, I. Redondo, L. Romero, S. Sánchez Navas, M.S. Soares, A. Triossi, C. Willmott

Universidad Autónoma de Madrid, Madrid, Spain

C. Albajar, J.F. de Trocóniz, R. Reyes-Almanza

Universidad de Oviedo, Instituto Universitario de Ciencias y Tecnologías Espaciales de Asturias (ICTEA), Oviedo, Spain

B. Alvarez Gonzalez, J. Cuevas, C. Erice, J. Fernandez Menendez, S. Folgueras, I. Gonzalez Caballero, J.R. González Fernández, E. Palencia Cortezon, V. Rodríguez Bouza, S. Sanchez Cruz

Instituto de Física de Cantabria (IFCA), CSIC-Universidad de Cantabria, Santander, Spain

I.J. Cabrillo, A. Calderon, B. Chazin Quero, J. Duarte Campderros, M. Fernandez, P.J. Fernández Manteca, A. García Alonso, G. Gomez, C. Martinez Rivero, P. Martinez Ruiz del Arbol, F. Matorras, J. Piedra Gomez, C. Prieels, T. Rodrigo, A. Ruiz-Jimeno, L. Russo $^{47}$, L. Scodellaro, I. Vila, J.M. Vizan Garcia

University of Colombo, Colombo, Sri Lanka

K. Malagalage

University of Ruhuna, Department of Physics, Matara, Sri Lanka

W.G.D. Dharmaratna, N. Wickramage 


\section{CERN, European Organization for Nuclear Research, Geneva, Switzerland}

D. Abbaneo, B. Akgun, E. Auffray, G. Auzinger, J. Baechler, P. Baillon, A.H. Ball, D. Barney, J. Bendavid, M. Bianco, A. Bocci, P. Bortignon, E. Bossini, C. Botta, E. Brondolin, T. Camporesi, A. Caratelli, G. Cerminara, E. Chapon, G. Cucciati, D. d'Enterria, A. Dabrowski, N. Daci, V. Daponte, A. David, O. Davignon, A. De Roeck, M. Deile, M. Dobson, M. Dünser, N. Dupont, A. Elliott-Peisert, N. Emriskova, F. Fallavollita ${ }^{48}$, D. Fasanella, S. Fiorendi, G. Franzoni, J. Fulcher, W. Funk, S. Giani, D. Gigi, A. Gilbert, K. Gill, F. Glege, L. Gouskos, M. Gruchala, M. Guilbaud, D. Gulhan, J. Hegeman, C. Heidegger, Y. Iiyama, V. Innocente, T. James, P. Janot, O. Karacheban ${ }^{20}$, J. Kaspar, J. Kieseler, M. Krammer ${ }^{1}$, N. Kratochwil, C. Lange, P. Lecoq, C. Lourenço, L. Malgeri, M. Mannelli, A. Massironi, F. Meijers, J.A. Merlin, S. Mersi, E. Meschi, F. Moortgat, M. Mulders, J. Ngadiuba, J. Niedziela, S. Nourbakhsh, S. Orfanelli, L. Orsini, F. Pantaleo ${ }^{17}$, L. Pape, E. Perez, M. Peruzzi, A. Petrilli, G. Petrucciani, A. Pfeiffer, M. Pierini, F.M. Pitters, D. Rabady, A. Racz, M. Rieger, M. Rovere, H. Sakulin, J. SalfeldNebgen, C. Schäfer, C. Schwick, M. Selvaggi, A. Sharma, P. Silva, W. Snoeys, P. Sphicas ${ }^{49}$, J. Steggemann, S. Summers, V.R. Tavolaro, D. Treille, A. Tsirou, G.P. Van Onsem, A. Vartak, M. Verzetti, W.D. Zeuner

\section{Paul Scherrer Institut, Villigen, Switzerland}

L. Caminada ${ }^{50}$, K. Deiters, W. Erdmann, R. Horisberger, Q. Ingram, H.C. Kaestli, D. Kotlinski, U. Langenegger, T. Rohe, S.A. Wiederkehr

ETH Zurich - Institute for Particle Physics and Astrophysics (IPA), Zurich, Switzerland

M. Backhaus, P. Berger, N. Chernyavskaya, G. Dissertori, M. Dittmar, M. Donegà, C. Dorfer, T.A. Gómez Espinosa, C. Grab, D. Hits, W. Lustermann, R.A. Manzoni, M.T. Meinhard, F. Micheli, P. Musella, F. Nessi-Tedaldi, F. Pauss, G. Perrin, L. Perrozzi, S. Pigazzini, M.G. Ratti, M. Reichmann, C. Reissel, T. Reitenspiess, B. Ristic, D. Ruini, D.A. Sanz Becerra, M. Schönenberger, L. Shchutska, M.L. Vesterbacka Olsson, R. Wallny, D.H. Zhu

\section{Universität Zürich, Zurich, Switzerland}

T.K. Aarrestad, C. Amsler ${ }^{51}$, D. Brzhechko, M.F. Canelli, A. De Cosa, R. Del Burgo, B. Kilminster, S. Leontsinis, V.M. Mikuni, I. Neutelings, G. Rauco, P. Robmann, K. Schweiger, C. Seitz, Y. Takahashi, S. Wertz, A. Zucchetta

\section{National Central University, Chung-Li, Taiwan}

T.H. Doan, C.M. Kuo, W. Lin, A. Roy, S.S. Yu

\section{National Taiwan University (NTU), Taipei, Taiwan}

P. Chang, Y. Chao, K.F. Chen, P.H. Chen, W.-S. Hou, Y.y. Li, R.-S. Lu, E. Paganis, A. Psallidas, A. Steen

Chulalongkorn University, Faculty of Science, Department of Physics, Bangkok, Thailand

B. Asavapibhop, C. Asawatangtrakuldee, N. Srimanobhas, N. Suwonjandee 
Çukurova University, Physics Department, Science and Art Faculty, Adana, Turkey

A. Bat, F. Boran, A. Celik ${ }^{52}$, S. Cerci ${ }^{53}$, S. Damarseckin ${ }^{54}$, Z.S. Demiroglu, F. Dolek, C. Dozen ${ }^{55}$, I. Dumanoglu, G. Gokbulut, EmineGurpinar Guler ${ }^{56}$, Y. Guler, I. Hos ${ }^{57}$, C. Isik, E.E. Kangal ${ }^{58}$, O. Kara, A. Kayis Topaksu, U. Kiminsu, G. Onengut, K. Ozdemir ${ }^{59}$, S. Ozturk ${ }^{60}$, A.E. Simsek, D. Sunar Cerci ${ }^{53}$, U.G. Tok, S. Turkcapar, I.S. Zorbakir, C. Zorbilmez

Middle East Technical University, Physics Department, Ankara, Turkey B. Isildak ${ }^{61}$, G. Karapinar ${ }^{62}$, M. Yalvac

Bogazici University, Istanbul, Turkey

I.O. Atakisi, E. Gülmez, M. Kaya ${ }^{63}$, O. Kaya ${ }^{64}$, Ö. Özçelik, S. Tekten, E.A. Yetkin ${ }^{65}$

Istanbul Technical University, Istanbul, Turkey

A. Cakir, K. Cankocak, Y. Komurcu, S. Sen ${ }^{66}$

Istanbul University, Istanbul, Turkey

B. Kaynak, S. Ozkorucuklu

Institute for Scintillation Materials of National Academy of Science of Ukraine, Kharkov, Ukraine

B. Grynyov

National Scientific Center, Kharkov Institute of Physics and Technology, Kharkov, Ukraine

L. Levchuk

University of Bristol, Bristol, United Kingdom

E. Bhal, S. Bologna, J.J. Brooke, D. Burns ${ }^{67}$, E. Clement, D. Cussans, H. Flacher, J. Goldstein, G.P. Heath, H.F. Heath, L. Kreczko, B. Krikler, S. Paramesvaran, B. Penning, T. Sakuma, S. Seif El Nasr-Storey, V.J. Smith, J. Taylor, A. Titterton

Rutherford Appleton Laboratory, Didcot, United Kingdom

K.W. Bell, A. Belyaev ${ }^{68}$, C. Brew, R.M. Brown, D.J.A. Cockerill, J.A. Coughlan, K. Harder, S. Harper, J. Linacre, K. Manolopoulos, D.M. Newbold, E. Olaiya, D. Petyt, T. Reis, T. Schuh, C.H. Shepherd-Themistocleous, A. Thea, I.R. Tomalin, T. Williams, W.J. Womersley

\section{Imperial College, London, United Kingdom}

R. Bainbridge, P. Bloch, J. Borg, S. Breeze, O. Buchmuller, A. Bundock, GurpreetSingh CHAHAL ${ }^{69}$, D. Colling, P. Dauncey, G. Davies, M. Della Negra, R. Di Maria, P. Everaerts, G. Hall, G. Iles, M. Komm, C. Laner, L. Lyons, A.-M. Magnan, S. Malik, A. Martelli, V. Milosevic, A. Morton, J. Nash ${ }^{70}$, V. Palladino, M. Pesaresi, D.M. Raymond, A. Richards, A. Rose, E. Scott, C. Seez, A. Shtipliyski, M. Stoye, T. Strebler, A. Tapper, K. Uchida, T. Virdee ${ }^{17}$, N. Wardle, D. Winterbottom, J. Wright, A.G. Zecchinelli, S.C. Zenz 
Brunel University, Uxbridge, United Kingdom

J.E. Cole, P.R. Hobson, A. Khan, P. Kyberd, C.K. Mackay, I.D. Reid, L. Teodorescu, S. Zahid

Baylor University, Waco, U.S.A.

K. Call, B. Caraway, J. Dittmann, K. Hatakeyama, C. Madrid, B. McMaster, N. Pastika, C. Smith

Catholic University of America, Washington, DC, U.S.A.

R. Bartek, A. Dominguez, R. Uniyal, A.M. Vargas Hernandez

The University of Alabama, Tuscaloosa, U.S.A.

A. Buccilli, S.I. Cooper, C. Henderson, P. Rumerio, C. West

Boston University, Boston, U.S.A.

A. Albert, D. Arcaro, Z. Demiragli, D. Gastler, C. Richardson, J. Rohlf, D. Sperka, I. Suarez, L. Sulak, D. Zou

Brown University, Providence, U.S.A.

G. Benelli, B. Burkle, X. Coubez ${ }^{18}$, D. Cutts, Y.t. Duh, M. Hadley, U. Heintz, J.M. $\operatorname{Hogan}^{71}$, K.H.M. Kwok, E. Laird, G. Landsberg, K.T. Lau, J. Lee, Z. Mao, M. Narain, S. Sagir ${ }^{72}$, R. Syarif, E. Usai, D. Yu, W. Zhang

University of California, Davis, Davis, U.S.A.

R. Band, C. Brainerd, R. Breedon, M. Calderon De La Barca Sanchez, M. Chertok, J. Conway, R. Conway, P.T. Cox, R. Erbacher, C. Flores, G. Funk, F. Jensen, W. Ko, O. Kukral, R. Lander, M. Mulhearn, D. Pellett, J. Pilot, M. Shi, D. Taylor, K. Tos, M. Tripathi, Z. Wang, F. Zhang

University of California, Los Angeles, U.S.A.

M. Bachtis, C. Bravo, R. Cousins, A. Dasgupta, A. Florent, J. Hauser, M. Ignatenko, N. Mccoll, W.A. Nash, S. Regnard, D. Saltzberg, C. Schnaible, B. Stone, V. Valuev

University of California, Riverside, Riverside, U.S.A.

K. Burt, Y. Chen, R. Clare, J.W. Gary, S.M.A. Ghiasi Shirazi, G. Hanson, G. Karapostoli, E. Kennedy, O.R. Long, M. Olmedo Negrete, M.I. Paneva, W. Si, L. Wang, S. Wimpenny, B.R. Yates, Y. Zhang

University of California, San Diego, La Jolla, U.S.A.

J.G. Branson, P. Chang, S. Cittolin, S. Cooperstein, N. Deelen, M. Derdzinski, R. Gerosa, D. Gilbert, B. Hashemi, D. Klein, V. Krutelyov, J. Letts, M. Masciovecchio, S. May, S. Padhi, M. Pieri, V. Sharma, M. Tadel, F. Würthwein, A. Yagil, G. Zevi Della Porta

University of California, Santa Barbara - Department of Physics, Santa Barbara, U.S.A.

N. Amin, R. Bhandari, C. Campagnari, M. Citron, V. Dutta, M. Franco Sevilla, J. Incandela, B. Marsh, H. Mei, A. Ovcharova, H. Qu, J. Richman, U. Sarica, D. Stuart, S. Wang 
California Institute of Technology, Pasadena, U.S.A.

D. Anderson, A. Bornheim, O. Cerri, I. Dutta, J.M. Lawhorn, N. Lu, J. Mao, H.B. Newman, T.Q. Nguyen, J. Pata, M. Spiropulu, J.R. Vlimant, S. Xie, Z. Zhang, R.Y. Zhu

Carnegie Mellon University, Pittsburgh, U.S.A.

M.B. Andrews, T. Ferguson, T. Mudholkar, M. Paulini, M. Sun, I. Vorobiev, M. Weinberg

University of Colorado Boulder, Boulder, U.S.A.

J.P. Cumalat, W.T. Ford, E. MacDonald, T. Mulholland, R. Patel, A. Perloff, K. Stenson, K.A. Ulmer, S.R. Wagner

Cornell University, Ithaca, U.S.A.

J. Alexander, Y. Cheng, J. Chu, A. Datta, A. Frankenthal, K. Mcdermott, J.R. Patterson,

D. Quach, A. Ryd, S.M. Tan, Z. Tao, J. Thom, P. Wittich, M. Zientek

Fermi National Accelerator Laboratory, Batavia, U.S.A.

S. Abdullin, M. Albrow, M. Alyari, G. Apollinari, A. Apresyan, A. Apyan, S. Banerjee, L.A.T. Bauerdick, A. Beretvas, D. Berry, J. Berryhill, P.C. Bhat, K. Burkett, J.N. Butler, A. Canepa, G.B. Cerati, H.W.K. Cheung, F. Chlebana, M. Cremonesi, J. Duarte, V.D. Elvira, J. Freeman, Z. Gecse, E. Gottschalk, L. Gray, D. Green, S. Grünendahl, O. Gutsche, AllisonReinsvold Hall, J. Hanlon, R.M. Harris, S. Hasegawa, R. Heller, J. Hirschauer, B. Jayatilaka, S. Jindariani, M. Johnson, U. Joshi, T. Klijnsma, B. Klima, M.J. Kortelainen, B. Kreis, S. Lammel, J. Lewis, D. Lincoln, R. Lipton, M. Liu, T. Liu, J. Lykken, K. Maeshima, J.M. Marraffino, D. Mason, P. McBride, P. Merkel, S. Mrenna, S. Nahn, V. O'Dell, V. Papadimitriou, K. Pedro, C. Pena, G. Rakness, F. Ravera, L. Ristori, B. Schneider, E. Sexton-Kennedy, N. Smith, A. Soha, W.J. Spalding, L. Spiegel, S. Stoynev, J. Strait, N. Strobbe, L. Taylor, S. Tkaczyk, N.V. Tran, L. Uplegger, E.W. Vaandering, C. Vernieri, R. Vidal, M. Wang, H.A. Weber

University of Florida, Gainesville, U.S.A.

D. Acosta, P. Avery, D. Bourilkov, A. Brinkerhoff, L. Cadamuro, A. Carnes, V. Cherepanov, F. Errico, R.D. Field, S.V. Gleyzer, D. Guerrero, B.M. Joshi, M. Kim, J. Konigsberg, A. Korytov, K.H. Lo, P. Ma, K. Matchev, N. Menendez, G. Mitselmakher, D. Rosenzweig, K. Shi, J. Wang, X. Zuo

Florida International University, Miami, U.S.A.

Y.R. Joshi

Florida State University, Tallahassee, U.S.A.

T. Adams, A. Askew, S. Hagopian, V. Hagopian, K.F. Johnson, R. Khurana, T. Kolberg, G. Martinez, T. Perry, H. Prosper, C. Schiber, R. Yohay, J. Zhang

Florida Institute of Technology, Melbourne, U.S.A.

M.M. Baarmand, M. Hohlmann, D. Noonan, M. Rahmani, M. Saunders, F. Yumiceva

University of Illinois at Chicago (UIC), Chicago, U.S.A.

M.R. Adams, L. Apanasevich, R.R. Betts, R. Cavanaugh, X. Chen, S. Dittmer, O. Evdokimov, C.E. Gerber, D.A. Hangal, D.J. Hofman, K. Jung, C. Mills, T. Roy, M.B. Tonjes, N. Varelas, J. Viinikainen, H. Wang, X. Wang, Z. Wu 
The University of Iowa, Iowa City, U.S.A.

M. Alhusseini, B. Bilki ${ }^{56}$, W. Clarida, K. Dilsiz ${ }^{73}$, S. Durgut, R.P. Gandrajula, M. Haytmyradov, V. Khristenko, O.K. Köseyan, J.-P. Merlo, A. Mestvirishvili ${ }^{74}$, A. Moeller, J. Nachtman, H. Ogul ${ }^{75}$, Y. Onel, F. Ozok ${ }^{76}$, A. Penzo, C. Snyder, E. Tiras, J. Wetzel

Johns Hopkins University, Baltimore, U.S.A.

B. Blumenfeld, A. Cocoros, N. Eminizer, A.V. Gritsan, W.T. Hung, S. Kyriacou, P. Maksimovic, J. Roskes, M. Swartz

The University of Kansas, Lawrence, U.S.A.

C. Baldenegro Barrera, P. Baringer, A. Bean, S. Boren, J. Bowen, A. Bylinkin,

T. Isidori, S. Khalil, J. King, G. Krintiras, A. Kropivnitskaya, C. Lindsey, D. Majumder,

W. Mcbrayer, N. Minafra, M. Murray, C. Rogan, C. Royon, S. Sanders, E. Schmitz, J.D. Tapia Takaki, Q. Wang, J. Williams, G. Wilson

Kansas State University, Manhattan, U.S.A.

S. Duric, A. Ivanov, K. Kaadze, D. Kim, Y. Maravin, D.R. Mendis, T. Mitchell, A. Modak, A. Mohammadi

Lawrence Livermore National Laboratory, Livermore, U.S.A.

F. Rebassoo, D. Wright

University of Maryland, College Park, U.S.A.

A. Baden, O. Baron, A. Belloni, S.C. Eno, Y. Feng, N.J. Hadley, S. Jabeen, G.Y. Jeng, R.G. Kellogg, J. Kunkle, A.C. Mignerey, S. Nabili, F. Ricci-Tam, M. Seidel, Y.H. Shin, A. Skuja, S.C. Tonwar, K. Wong

Massachusetts Institute of Technology, Cambridge, U.S.A.

D. Abercrombie, B. Allen, A. Baty, R. Bi, S. Brandt, W. Busza, I.A. Cali, M. D'Alfonso,

G. Gomez Ceballos, M. Goncharov, P. Harris, D. Hsu, M. Hu, M. Klute, D. Kovalskyi, Y.-J. Lee, P.D. Luckey, B. Maier, A.C. Marini, C. Mcginn, C. Mironov, S. Narayanan, X. Niu, C. Paus, D. Rankin, C. Roland, G. Roland, Z. Shi, G.S.F. Stephans, K. Sumorok, K. Tatar, D. Velicanu, J. Wang, T.W. Wang, B. Wyslouch

University of Minnesota, Minneapolis, U.S.A.

R.M. Chatterjee, A. Evans, S. Guts ${ }^{\dagger}$, P. Hansen, J. Hiltbrand, Sh. Jain, Y. Kubota, Z. Lesko, J. Mans, M. Revering, R. Rusack, R. Saradhy, N. Schroeder, M.A. Wadud

University of Mississippi, Oxford, U.S.A.

J.G. Acosta, S. Oliveros

University of Nebraska-Lincoln, Lincoln, U.S.A.

K. Bloom, S. Chauhan, D.R. Claes, C. Fangmeier, L. Finco, F. Golf, R. Kamalieddin, I. Kravchenko, J.E. Siado, G.R. Snow ${ }^{\dagger}$, B. Stieger, W. Tabb

State University of New York at Buffalo, Buffalo, U.S.A.

G. Agarwal, C. Harrington, I. Iashvili, A. Kharchilava, C. McLean, D. Nguyen, A. Parker, J. Pekkanen, S. Rappoccio, B. Roozbahani 
Northeastern University, Boston, U.S.A.

G. Alverson, E. Barberis, C. Freer, Y. Haddad, A. Hortiangtham, G. Madigan, B. Marzocchi, D.M. Morse, T. Orimoto, L. Skinnari, A. Tishelman-Charny, T. Wamorkar, B. Wang, A. Wisecarver, D. Wood

Northwestern University, Evanston, U.S.A.

S. Bhattacharya, J. Bueghly, T. Gunter, K.A. Hahn, N. Odell, M.H. Schmitt, K. Sung, M. Trovato, M. Velasco

University of Notre Dame, Notre Dame, U.S.A.

R. Bucci, N. Dev, R. Goldouzian, M. Hildreth, K. Hurtado Anampa, C. Jessop, D.J. Karmgard, K. Lannon, W. Li, N. Loukas, N. Marinelli, I. Mcalister, F. Meng, C. Mueller, Y. Musienko ${ }^{38}$, M. Planer, R. Ruchti, P. Siddireddy, G. Smith, S. Taroni, M. Wayne, A. Wightman, M. Wolf, A. Woodard

The Ohio State University, Columbus, U.S.A.

J. Alimena, B. Bylsma, L.S. Durkin, B. Francis, C. Hill, W. Ji, A. Lefeld, T.Y. Ling, B.L. Winer

Princeton University, Princeton, U.S.A.

G. Dezoort, P. Elmer, J. Hardenbrook, N. Haubrich, S. Higginbotham, A. Kalogeropoulos, S. Kwan, D. Lange, M.T. Lucchini, J. Luo, D. Marlow, K. Mei, I. Ojalvo, J. Olsen, C. Palmer, P. Piroué, D. Stickland, C. Tully, Z. Wang

University of Puerto Rico, Mayaguez, U.S.A.

S. Malik, S. Norberg

Purdue University, West Lafayette, U.S.A.

A. Barker, V.E. Barnes, S. Das, L. Gutay, M. Jones, A.W. Jung, A. Khatiwada, B. Mahakud, D.H. Miller, G. Negro, N. Neumeister, C.C. Peng, S. Piperov, H. Qiu, J.F. Schulte, N. Trevisani, F. Wang, R. Xiao, W. Xie

Purdue University Northwest, Hammond, U.S.A.

T. Cheng, J. Dolen, N. Parashar

Rice University, Houston, U.S.A.

U. Behrens, K.M. Ecklund, S. Freed, F.J.M. Geurts, M. Kilpatrick, Arun Kumar, W. Li, B.P. Padley, R. Redjimi, J. Roberts, J. Rorie, W. Shi, A.G. Stahl Leiton, Z. Tu, A. Zhang

University of Rochester, Rochester, U.S.A.

A. Bodek, P. de Barbaro, R. Demina, J.L. Dulemba, C. Fallon, T. Ferbel, M. Galanti, A. Garcia-Bellido, O. Hindrichs, A. Khukhunaishvili, E. Ranken, R. Taus

Rutgers, The State University of New Jersey, Piscataway, U.S.A.

B. Chiarito, J.P. Chou, A. Gandrakota, Y. Gershtein, E. Halkiadakis, A. Hart, M. Heindl,

E. Hughes, S. Kaplan, I. Laflotte, A. Lath, R. Montalvo, K. Nash, M. Osherson, H. Saka,

S. Salur, S. Schnetzer, S. Somalwar, R. Stone, S. Thomas 
University of Tennessee, Knoxville, U.S.A.

H. Acharya, A.G. Delannoy, S. Spanier

Texas A\&M University, College Station, U.S.A.

O. Bouhali ${ }^{77}$, M. Dalchenko, M. De Mattia, A. Delgado, S. Dildick, R. Eusebi, J. Gilmore,

T. Huang, T. Kamon ${ }^{78}$, H. Kim, S. Luo, S. Malhotra, D. Marley, R. Mueller, D. Overton,

L. Perniè, D. Rathjens, A. Safonov

Texas Tech University, Lubbock, U.S.A.

N. Akchurin, J. Damgov, F. De Guio, V. Hegde, S. Kunori, K. Lamichhane, S.W. Lee, T. Mengke, S. Muthumuni, T. Peltola, S. Undleeb, I. Volobouev, Z. Wang, A. Whitbeck

Vanderbilt University, Nashville, U.S.A.

S. Greene, A. Gurrola, R. Janjam, W. Johns, C. Maguire, A. Melo, H. Ni, K. Padeken, F. Romeo, P. Sheldon, S. Tuo, J. Velkovska, M. Verweij

University of Virginia, Charlottesville, U.S.A.

M.W. Arenton, P. Barria, B. Cox, G. Cummings, J. Hakala, R. Hirosky, M. Joyce, A. Ledovskoy, C. Neu, B. Tannenwald, Y. Wang, E. Wolfe, F. Xia

Wayne State University, Detroit, U.S.A.

R. Harr, P.E. Karchin, N. Poudyal, J. Sturdy, P. Thapa

University of Wisconsin - Madison, Madison, WI, U.S.A.

T. Bose, J. Buchanan, C. Caillol, D. Carlsmith, S. Dasu, I. De Bruyn, L. Dodd, C. Galloni, H. He, M. Herndon, A. Hervé, U. Hussain, P. Klabbers, A. Lanaro, A. Loeliger, K. Long, R. Loveless, J. Madhusudanan Sreekala, D. Pinna, T. Ruggles, A. Savin, V. Sharma, W.H. Smith, D. Teague, S. Trembath-reichert, N. Woods

$\dagger$ : Deceased

1: Also at Vienna University of Technology, Vienna, Austria

2: Also at IRFU, CEA, Université Paris-Saclay, Gif-sur-Yvette, France

3: Also at Universidade Estadual de Campinas, Campinas, Brazil

4: Also at Federal University of Rio Grande do Sul, Porto Alegre, Brazil

5: Also at UFMS, Nova Andradina, Brazil

6: Also at Universidade Federal de Pelotas, Pelotas, Brazil

7: Also at Université Libre de Bruxelles, Bruxelles, Belgium

8: Also at University of Chinese Academy of Sciences, Beijing, China

9: Also at Institute for Theoretical and Experimental Physics named by A.I. Alikhanov of NRC 'Kurchatov Institute', Moscow, Russia

10: Also at Joint Institute for Nuclear Research, Dubna, Russia

11: Also at Helwan University, Cairo, Egypt

12: Now at Zewail City of Science and Technology, Zewail, Egypt

13: Also at Purdue University, West Lafayette, U.S.A.

14: Also at Université de Haute Alsace, Mulhouse, France

15: Also at Tbilisi State University, Tbilisi, Georgia

16: Also at Erzincan Binali Yildirim University, Erzincan, Turkey

17: Also at CERN, European Organization for Nuclear Research, Geneva, Switzerland 
18: Also at RWTH Aachen University, III. Physikalisches Institut A, Aachen, Germany

19: Also at University of Hamburg, Hamburg, Germany

20: Also at Brandenburg University of Technology, Cottbus, Germany

21: Also at Institute of Physics, University of Debrecen, Debrecen, Hungary, Debrecen, Hungary

22: Also at Institute of Nuclear Research ATOMKI, Debrecen, Hungary

23: Also at MTA-ELTE Lendület CMS Particle and Nuclear Physics Group, Eötvös Loránd University, Budapest, Hungary, Budapest, Hungary

24: Also at IIT Bhubaneswar, Bhubaneswar, India, Bhubaneswar, India

25: Also at Institute of Physics, Bhubaneswar, India

26: Also at Shoolini University, Solan, India

27: Also at University of Hyderabad, Hyderabad, India

28: Also at University of Visva-Bharati, Santiniketan, India

29: Also at Isfahan University of Technology, Isfahan, Iran

30: Now at INFN Sezione di Bari ${ }^{a}$, Università di Bari ${ }^{b}$, Politecnico di Bari ${ }^{c}$, Bari, Italy

31: Also at Italian National Agency for New Technologies, Energy and Sustainable Economic Development, Bologna, Italy

32: Also at Centro Siciliano di Fisica Nucleare e di Struttura Della Materia, Catania, Italy

33: Also at Scuola Normale e Sezione dell'INFN, Pisa, Italy

34: Also at Riga Technical University, Riga, Latvia, Riga, Latvia

35: Also at Malaysian Nuclear Agency, MOSTI, Kajang, Malaysia

36: Also at Consejo Nacional de Ciencia y Tecnología, Mexico City, Mexico

37: Also at Warsaw University of Technology, Institute of Electronic Systems, Warsaw, Poland

38: Also at Institute for Nuclear Research, Moscow, Russia

39: Now at National Research Nuclear University 'Moscow Engineering Physics Institute' (MEPhI), Moscow, Russia

40: Also at St. Petersburg State Polytechnical University, St. Petersburg, Russia

41: Also at University of Florida, Gainesville, U.S.A.

42: Also at Imperial College, London, United Kingdom

43: Also at P.N. Lebedev Physical Institute, Moscow, Russia

44: Also at California Institute of Technology, Pasadena, U.S.A.

45: Also at Budker Institute of Nuclear Physics, Novosibirsk, Russia

46: Also at Faculty of Physics, University of Belgrade, Belgrade, Serbia

47: Also at Università degli Studi di Siena, Siena, Italy

48: Also at INFN Sezione di Pavia ${ }^{a}$, Università di Pavia ${ }^{b}$, Pavia, Italy, Pavia, Italy

49: Also at National and Kapodistrian University of Athens, Athens, Greece

50: Also at Universität Zürich, Zurich, Switzerland

51: Also at Stefan Meyer Institute for Subatomic Physics, Vienna, Austria, Vienna, Austria

52: Also at Burdur Mehmet Akif Ersoy University, BURDUR, Turkey

53: Also at Adiyaman University, Adiyaman, Turkey

54: Also at Şırnak University, Sirnak, Turkey

55: Also at Tsinghua University, Beijing, China

56: Also at Beykent University, Istanbul, Turkey, Istanbul, Turkey

57: Also at Istanbul Aydin University, Application and Research Center for Advanced Studies (App. \& Res. Cent. for Advanced Studies), Istanbul, Turkey

58: Also at Mersin University, Mersin, Turkey

59: Also at Piri Reis University, Istanbul, Turkey

60: Also at Gaziosmanpasa University, Tokat, Turkey

61: Also at Ozyegin University, Istanbul, Turkey 
62: Also at Izmir Institute of Technology, Izmir, Turkey

63: Also at Marmara University, Istanbul, Turkey

64: Also at Kafkas University, Kars, Turkey

65: Also at Istanbul Bilgi University, Istanbul, Turkey

66: Also at Hacettepe University, Ankara, Turkey

67: Also at Vrije Universiteit Brussel, Brussel, Belgium

68: Also at School of Physics and Astronomy, University of Southampton, Southampton, United Kingdom

69: Also at IPPP Durham University, Durham, United Kingdom

70: Also at Monash University, Faculty of Science, Clayton, Australia

71: Also at Bethel University, St. Paul, Minneapolis, U.S.A., St. Paul, U.S.A.

72: Also at Karamanoğlu Mehmetbey University, Karaman, Turkey

73: Also at Bingol University, Bingol, Turkey

74: Also at Georgian Technical University, Tbilisi, Georgia

75: Also at Sinop University, Sinop, Turkey

76: Also at Mimar Sinan University, Istanbul, Istanbul, Turkey

77: Also at Texas A\&M University at Qatar, Doha, Qatar

78: Also at Kyungpook National University, Daegu, Korea, Daegu, Korea 